\title{
El bienestar de la población de los estados de México bajo un enfoque de multidimensionalidad
}

\author{
Amilcar O. Fernández Domínguez \\ y Denise Gómez Hernández
}

\section{Resumen}

En el presente artículo se adapta un índice multidimensional de bienestar de la población de los estados mexicanos sobre la base de las recomendaciones de la Comisión sobre la Medición del Desempeño Económico y el Progreso Social. Los aportes de este estudio se resumen en tres puntos importantes: i) se emplea el análisis de factores de componentes principales para permitir que las dimensiones tengan diferente ponderación, ii) se considera la desigualdad del bienestar material de la población dentro de cada estado, y iii) se contemplan datos representativos a nivel estatal de todas las dimensiones. Los resultados muestran que las dimensiones relacionadas con el bienestar objetivo tienen mayor ponderación que las dimensiones de bienestar subjetivo, y que las diferencias en la ponderación de las dimensiones e indicadores utilizados son más importantes que su número y características.

\section{Palabras clave}

Bienestar social, medición, metodología estadística, desarrollo económico, desarrollo social, indicadores del desarrollo, México

\section{Clasificación JEL}

I31, O54, C38, C43

\section{Autores}

Amilcar Orlian Fernández Domínguez es Profesor e Investigador en la Facultad de Economía Internacional de la Universidad Autónoma de Chihuahua (México). Correo electrónico: afernand@uach.mx.

Denise Gómez Hernández es Profesora e Investigadora en la Facultad de Contaduría y Administración de la Universidad Autónoma de Querétaro (México). Correo electrónico: denise.gomez@uaq.mx. 


\section{Introducción}

El concepto de multidimensionalidad del bienestar ha cobrado importancia recientemente a pesar de haber estado implícito en la literatura desde hace tiempo. Por ejemplo, Hicks (2002) y Nafziger (2005) citan que hay una serie de funciones básicas relacionadas con el bienestar, como una nutrición adecuada, la ausencia de mortalidad prematura, la capacidad de mostrarse en público sin vergüenza u otros. Primordialmente el enfoque ha estado en los aspectos materiales del bienestar; al respecto, tuvieron gran auge algunas medidas objetivas como el índice de desarrollo humano o la disminución de la población en situación de pobreza. Sin embargo, últimamente se ha enfatizado que la multidimensionalidad del bienestar abarca, además, la medición de aspectos inmateriales (Bérenger y Verdier-Chouchane, 2007; Aiginger y Firgo, 2015; Arita, 2005), lo que ha centrado una mayor atención en la importancia de incorporar un enfoque de bienestar subjetivo en el análisis.

Tocante al caso mexicano, el objetivo de incrementar los estándares de vida y bienestar de sus residentes es evidente en el Plan Nacional de Desarrollo 2013-2018 (México, Gobierno de, 2013). Al respecto, la OCDE (2014) aclara que para implementar políticas públicas adecuadas, es esencial contar con indicadores que reflejen convenientemente el nivel de bienestar de las poblaciones. Así, el tema gira en torno a dos cuestionamientos: saber quiénes tienen mayor bienestar y por qué. En cuanto a lo segundo, en la literatura se encuentran diversas concepciones y teorías respecto a qué es el bienestar y cuáles son sus determinantes, que abarcan desde los conceptos de eudemonía de los filósofos griegos hasta los enfoques más recientes de capacidades y bienestar subjetivo. El informe elaborado por Stiglitz, Sen y Fitoussi (2009) para la Comisión sobre la Medición del Desempeño Económico y el Progreso Social sintetiza un marco teórico pertinente al análisis de la multidimensionalidad del bienestar; estos enfoques teóricos se abordan en el segundo apartado de este artículo.

Respecto al primer cuestionamiento, Stiglitz, Sen y Fitoussi (2009) argumentan que los factores que afectan el bienestar de una persona requieren datos tanto objetivos como subjetivos. Brindan una serie de recomendaciones, entre las que destaca la construcción de una medida resumida y única de bienestar, y sugieren que la consideración del bienestar aborde tres enfoques o concepciones teóricas prominentes en la literatura: la teoría de capacidades, la teoría de bienestar subjetivo y la teoría de asignaciones justas. En el mismo sentido, señalan indicadores relativos a diversas facetas o dimensiones que inciden en el bienestar, como el ingreso disponible, el consumo y la riqueza (pertenecientes a la dimensión del bienestar material), y otros correspondientes a la salud, la educación, las actividades personales, la participación política, las conexiones sociales, el medioambiente y la inseguridad.

Del mismo modo, Stiglitz, Sen y Fitoussi (2009) enfatizan en su informe la importancia de considerar algunos factores limitantes al crear un indicador compuesto de bienestar: la pérdida de información en materia de desigualdad producto del uso de promedios, la elección arbitraria de ponderadores y de la información sobre la contribución relativa de cada dimensión del bienestar y la interpretación de los cambios en el indicador a través del tiempo. Por otro lado, estos autores mencionan las ventajas de analizar datos a nivel individual en vez de a nivel estatal o regional (ya que, por ejemplo, esto permite explorar la inequidad).

La aplicación de estos aspectos al caso mexicano ha sido insuficiente. Algunos aportes han brindado indicadores para medir el bienestar en México; el más reciente y notable, por su enfoque multidimensional, es el de la Organización para la Cooperación y el Desarrollo Económicos (OCDE) (2015), que se basa en los Indicadores de Bienestar por entidad federativa del Instituto Nacional de Estadística y Geografía de México (INEGl) y cuyos fundamentos se encuentran en el Índice para una Vida Mejor de la OCDE. Sin embargo, este índice presenta algunas limitaciones en lo referente a los aspectos metodológicos mencionados por Stiglitz, Sen y Fitoussi (2009), pues se basa en el argumento de que todas las dimensiones tienen la misma importancia en determinar el bienestar general, no 
capta la desigualdad interior de cada estado ${ }^{1} \mathrm{y}$, aunque emplea datos de bienestar subjetivo, estos no son representativos a nivel estatal. El presente estudio busca cubrir estas carencias mediante la elaboración de un indicador que permita asignar distintas ponderaciones a cada dimensión según las recomendaciones del manual para construir indicadores compuestos provisto por la OCDE (2008)2, que otorgue mayor importancia a la desigualdad de acuerdo a Stiglitz, Sen y Fitoussi (2009) y que emplee datos representativos a nivel estatal ${ }^{3}$.

El documento se estructura como sigue: en el segundo apartado se abordan de manera breve las diversas concepciones y teorías relativas al bienestar; en el tercer apartado se explica la metodología basada en la OCDE (2008) seguida para obtener el índice de bienestar multidimensional; en el cuarto apartado se muestran los resultados obtenidos a partir de esta metodología y se adaptan otros indicadores para comparar las diferencias que surgen al considerar diversas dimensiones e indicadores, así como al permitir distintas ponderaciones, y, finalmente, en el quinto apartado se brindan algunas conclusiones.

\section{Concepciones y teorías del bienestar}

El concepto de bienestar es complejo y un tanto ambiguo, por lo que su discusión implica establecer en primer lugar una base de diversas teorías surgidas desde los conceptos de eudemonía y hedonismo de los griegos clásicos (Villatoro, 2012; Valencia y Cuervo, 1999). En el enfoque de los filósofos griegos, si bien se contempla que se requieren bienes materiales para lograr el bienestar, se hace especial hincapié en el aspecto inmaterial (faceta psicológica del hombre) para lograr la plenitud de la vida o el placer experiencial. El hecho de que en la filosofía clásica se enfatice la búsqueda de plenitud del ser humano vincula este enfoque con el del bienestar subjetivo abordado posteriormente; Bentham (2000) llegó incluso a argumentar que el principio de utilidad clásica implicaba una sujeción del individuo al placer y el dolor experimentado (y, por tanto, a la felicidad adquirida) a partir de sus acciones. Las críticas sobre la imposibilidad de este enfoque de utilidad para otorgar valores al bienestar de los individuos y poder realizar comparaciones llevó a los bienestaristas a identificar el bienestar sobre la base de las elecciones de las personas de entre un conjunto de alternativas (preferencia revelada) y a asignar un valor numérico al estado social mediante funciones de bienestar social (Villatoro, 2012).

Por su parte, el enfoque de bienestar utilitarista referente a la opulencia observa la situación en la que se encuentra un individuo con relación a su acceso a bienes y servicios; así, cuanto mayor sea el acceso a bienes y servicios - por medio de un mayor ingreso-, mayor será el bienestar del individuo. Sen (1984) explica que este enfoque define el bienestar de manera demasiado escueta y deja pasar elementos importantes al limitar el bienestar de un individuo al cumplimiento de deseos (es decir, el individuo obtiene bienestar en el consumismo).

Estos enfoques son criticados por el hecho de que no se califique el origen de los deseos que llevan a la toma de decisiones, es decir, por no especificar el valor intrínseco del bienestar. Por ejemplo, Rawls (1971) afirma que se podría lograr un gran bienestar (desde el punto de vista utilitarista) permitiendo el funcionamiento y resultados del crimen organizado, lo que conllevaría un

\footnotetext{
1 En el anexo A1 se aprecia que la construcción del índice captura la desigualdad interestatal, pero no necesariamente la existente entre la población de un estado. Por ejemplo, al utilizar el promedio del ingreso disponible por hogar, es posible observar el mismo valor en dos estados, aunque su distribución sea desigual.

2 El manual brinda una serie de herramientas analíticas y recomendaciones para la construcción de indicadores compuestos en 10 pasos; para el análisis multivariable y la ponderación, este estudio se enfoca en el análisis de factores de componentes principales y el alfa de Cronbach (los aspectos técnicos se abordan en el anexo A1).

3 El Instituto Nacional de Estadística y Geografía de México (INEGI) menciona que la división política de este país está organizada en 32 estados o entidades federativas; por tanto, en lo sucesivo se empleará indistintamente cualquiera de los dos términos.
} 
mayor movimiento económico además de una mayor violencia. Por ello, Rawls (1971) propone un bienestar basado en la justicia social: el bienestar se alcanzaría mediante la obtención de bienes primarios catalogados en naturales (como alimento o vestido) y sociales (como libertad, derechos, oportunidades, ingreso e instituciones relativas a la justicia). Así, este enfoque postula que el bienestar emana de una distribución más equitativa y plantea un análisis profundo de una serie de teorías de justicia, como la teoría de las asignaciones justas.

Con base en el criterio de Kolm (1997) de equidad en ausencia de envidia, la asignación justa eficiente más simple es aquella en la que a todos los individuos se les asigna el mismo conjunto de bienes; sin embargo, un mayor conocimiento de las características de la población y de los bienes disponibles permite realizar asignaciones justas, aunque sean diferentes para los distintos individuos (Thomson, 2016). En otras palabras, es posible asignar bienes diferentes a los individuos de una sociedad sin que queden peor de como estarían si se les asignara el mismo conjunto de bienes a todos. Aunque, en este sentido, la teoría de las asignaciones justas permite un juicio neutral, ya que cada individuo tiene un concepto de determinado bien, también presenta la dificultad de suponer que el bien tendrá realmente el efecto esperado en cada uno de los individuos (Wells, 2016).

Como alternativa a estas teorías surgen las referentes a las capacidades (Stiglitz, Sen y Fitoussi, 2009; Bérenger y Verdier-Chouchane, 2007), en que el bienestar no se da por la característica del bien consumido, como plantea la teoría utilitarista, o por la asignación y concepto que tenga el individuo del bien, como plantea la teoría de las asignaciones justas, sino por la libertad que obtiene la persona de su capacidad para hacer o ser (Sen, 1984); es decir, el bienestar proviene del funcionamiento que se deriva de la capacidad obtenida del bien (Plata, 1999). Según Sen (1984), el bienestar se relaciona con la libertad que tiene el individuo para poder funcionar de cierta manera; así, los bienes deben proporcionarle la capacidad de elegir su forma de funcionar (ser o hacer) y no solo de hacer cosas (Beckley, 2002). En este sentido, hay una serie de funciones básicas que otorgan bienestar al individuo, entre las que se encuentran contar con nutrición adecuada, no estar expuesto a una mortalidad prematura, poder aparecer en público sin vergüenza, ser feliz y ser libre (Hicks, 2002; Nafziger, 2005). De acuerdo a este enfoque, el bienestar va más allá de lo establecido en la teoría de utilidad, de ahí la creación del índice de desarrollo humano por parte del Programa de las Naciones Unidas para el Desarrollo (PNUD) (Bérenger y Verdier-Chouchane, 2007).

Otro grupo de autores aborda un enfoque más complejo, correspondiente a la teoría del bienestar subjetivo, en que se argumenta que el bienestar del individuo como ser humano depende de la propia percepción respecto a distintos ámbitos o dominios de vida, y en esta influyen tanto aspectos materiales (económicos) como no materiales (Bonini, 2008; Rojas, 2005, 2007; Lever, 2004). Dado que el bienestar implica elementos que no se pueden cuantificar, como los aspectos emocionales, se propone determinarlo mediante un indicador de satisfacción de vida o felicidad que se obtiene preguntando directamente al individuo cuán satisfecho o feliz se encuentra en su vida (Veenhoven, 2005).

De estas teorías destacan dos aspectos importantes: el bienestar es multidimensional (contiene aspectos subjetivos y objetivos), y la importancia de cada dimensión en el bienestar total es variable. Algunos autores (Villatoro, 2012; Krauss y Graham, 2013; Stiglitz, Sen y Fitoussi, 2009; Rojas, 2007), si bien no rechazan el enfoque de capacidades (o el aspecto objetivo del bienestar), señalan la necesidad de incorporar el ámbito de la subjetividad en los análisis de bienestar pese a la dificultad que ello implica. En efecto, resulta conveniente proponer una medición de bienestar que incorpore aspectos objetivos (como los niveles de educación, salud e ingresos considerados en el enfoque de capacidades) y subjetivos (percepción de satisfacción o felicidad), además de indagar sobre la participación de cada uno en la composición del bienestar general de los individuos.

Algunas publicaciones han abordado la multidimensionalidad del bienestar. Por ejemplo, Bérenger y Verdier-Chouchane (2007), en un estudio internacional, abordan parcialmente la 
multidimensionalidad del bienestar en 170 países mediante el uso del análisis de factores y la teoría de conjuntos difusos, pero solo analizan la contribución de la dimensión objetiva del bienestar (calidad de vida y estándares de vida). En otro estudio internacional en el que se contempla el caso mexicano, Benvin, Rivera y Tromben (2016) incorporan el recurso tiempo como una dimensión del bienestar, que, además de incidir directamente en este, está correlacionada con otras dimensiones; sin embargo, al igual que el estudio de Bérenger y Verdier-Chouchane (2007), su enfoque multidimensional del bienestar abarca únicamente aspectos objetivos.

Por otra parte, en un estudio local en México, Arita (2005) realiza un análisis multidimensional considerando aspectos subjetivos y objetivos del bienestar a nivel individual. Su estudio provee un primer acercamiento a la multidimensionalidad del bienestar en México como lo plantean Stiglitz, Sen y Fitoussi (2009): mediante el uso de un análisis de factores, brinda información sobre el porcentaje de variación del bienestar explicado por cada dimensión. Sin embargo, sus resultados no se pueden generalizar a toda la nación, pues solo contempla información sobre el bienestar de los residentes de la ciudad de Culiacán, en el estado de Sinaloa.

Finalmente, la OCDE (2015) ofrece un estudio más amplio, en el que también se considera la multidimensionalidad del bienestar en las entidades mexicanas. Se basa en el marco de su iniciativa Índice para una Vida Mejor (que toma como punto de partida las recomendaciones de Stiglitz, Sen y Fitoussi, 2009) y los estudios y bases de datos sobre bienestar regional de la misma OCDE y el INEGI. El estudio provee información reciente de doce dimensiones del bienestar, así como de un índice global de bienestar estatal. No obstante, los resultados no son del todo confiables si se tiene en cuenta la crítica y las recomendaciones respecto a la creación de indicadores de bienestar discutidas anteriormente.

En el estudio de la OCDE (2015) se presenta un índice de bienestar global para cada estado construido a partir del promedio y la varianza de índices de Mazziotta-Pareto ajustados (AMPI) obtenidos de cada dimensión del bienestar. Todos los indicadores considerados tienen la misma importancia o ponderación en la construcción del índice. Cada AMPI, a su vez, está construido a partir de la media y varianza de indicadores normalizados, como el promedio del ingreso disponible. Por tanto, mediante esta metodología es posible obtener el mismo valor de AMPI para una dimensión en dos estados que tengan distinta distribución ${ }^{4}$. Además, los datos referentes a los indicadores de bienestar subjetivos utilizados fueron tomados de una encuesta provista por el INEGI que no tenía representatividad estatal. Con base en lo anterior, puede argumentarse que, por un lado, este estudio de la OCDE otorga arbitrariamente la misma importancia a cada dimensión del bienestar, y, por otro, presta menor atención a la desigualdad del interior de cada estado, lo cual es relevante en un país como México.

\section{Metodología}

Para la creación del índice multidimensional de bienestar estatal (IMBE) se parte de las recomendaciones del manual para construir indicadores compuestos de la OCDE (2008) y del informe de Stiglitz, Sen y Fitoussi (2009). A partir de los lineamientos de la OCDE (2008) se establece la siguiente ecuación (1):

$$
I M B E_{i}=\sum W_{k}\left(\sum_{j k} X_{i j}\right)
$$

\footnotetext{
4 En un ejemplo hipotético, un estado cuyos miembros tengan todos un valor de 5 en algún indicador tendrá el mismo promedio estatal que un estado en que la mitad de los miembros tenga un valor de 0 y la otra mitad, de 10 en el mismo indicador (en ambos casos se obtendría un promedio estatal de 5).
} 
donde:

$I M B E_{i}$ se refiere al índice multidimensional de bienestar del estado $i$;

$W_{k}$ se refiere a la proporción de varianza del componente principal $k$ respecto a la varianza total de los componentes considerados en la construcción;

$x_{i j}$ se refiere al valor de cada indicador $j$ correspondiente al estado $i$, y

$w_{j k}$ se refiere a la ponderación normalizada correspondiente al indicador $j$ y el componente $k$.

El cálculo del $I M B E_{i}$ requiere determinar los valores de $W_{k}$ y $w_{j k}$, que se obtienen del análisis de factores de componentes principales. Sin embargo, en una primera etapa es necesario analizar si los indicadores $x_{i j}$ que se seleccionarán están relacionados entre sí, es decir, si explican conjuntamente la dimensión de bienestar que se está buscando determinar. Para ello se realiza un análisis exploratorio (gráfico) de dichos indicadores que permita tener una idea previa de las características y magnitud de las relaciones entre ellos. Considerando los factores limitantes indicados por Stiglitz, Sen y Fitoussi (2009), estos se reconocen utilizando microdatos a nivel individual para obtener información concerniente a la desigualdad, y se aplica la técnica de análisis de factores para permitir diferencias en los ponderadores de cada dimensión. Además, se emplea información de bienestar subjetivo medible y representativa a nivel estatal.

Se planteó la construcción del $I M B E_{i}$ a partir de las ocho dimensiones expuestas por Stiglitz, Sen y Fitoussi (2009). Estos autores sugieren una amplia lista de indicadores que considerar en cada dimensión; sin embargo, por el número relativamente bajo de observaciones (32 estados) y las restricciones estadísticas de los métodos empleados, se incorporaron en $x_{i j}$ los indicadores disponibles con el mayor aporte esperado de información respecto a cada dimensión. Los indicadores utilizados para la construcción del $I M B E_{i}$, enumerados en el cuadro 1, comprenden: la mediana del ingreso total (por ingresos y riqueza) corriente (inghmed), el ingreso disponible ajustado por el coeficiente de Gini estatal (ingaj), la mediana del gasto total anual del hogar ajustado por unidad de consumo (gpcmed), la media de la situación de salud declarada (situsalud), la media del acceso a la salud (accesalud), la media de la escolaridad acumulada (educ), la media de la satisfacción con la actividad que se realiza (satisactiv), la media de la libertad de decisión declarada (libertad), la media de la satisfacción con la vida social (satissocial), la contaminación del aire $\left(\mathrm{CO}_{2}\right)$, y la media de la satisfacción con la seguridad ciudadana (satissecure).

De acuerdo con la OCDE (2008), el análisis de factores de componentes principales permite formar un indicador compuesto que capture la mayor cantidad de información común de entre un grupo de variables, de manera que el indicador compuesto no dependa de la dimensionalidad de los datos. Por esta razón, después del análisis gráfico de los indicadores $x_{i j}$, en una segunda etapa se realiza el cálculo del coeficiente alfa de Cronbach, que permite medir la confiabilidad (una primera idea de la contribución) de cada indicador en el índice global de bienestar y, de esta forma, seleccionar los indicadores $x_{i j}$ que realmente aporten significativamente al $I M B E_{i}$ y que mantengan el principio de parsimonia. En una tercera etapa se realiza un análisis de correlaciones entre los indicadores $x_{i j}$, en el que se observa la multidimensionalidad del $I M B E_{i}$ y, posteriormente, en una cuarta etapa se realiza el análisis de componentes de factores principales (en el anexo A1 se incluyen algunas notas técnicas referentes a estas etapas).

Finalmente, en una quinta etapa se realiza una comparación del $I M B E_{i}$ con otros indicadores de bienestar. Esta comparación se puede observar en el cuadro 1: en la primera y segunda columnas se resaltan en negrita las dimensiones e indicadores que se consideran en el índice propuesto $I M B E_{i}$, mientras que en las siguientes columnas se muestran las dimensiones e indicadores de dos índices adicionales, obtenidos mediante la misma metodología de análisis de factores de componentes principales, pero adaptados, respectivamente, del enfoque de multidimensionalidad del Índice para una Vida Mejor de la OCDE (2015) ( $B L I_{i}$ ) y del enfoque recogido por Bérenger y Verdier-Chouchane (2007) ( $\left.S o L_{i}\right)$. 
Cuadro 1

Dimensiones e indicadores considerados en cada índice de bienestar

\begin{tabular}{|c|c|c|c|c|c|}
\hline \multicolumn{2}{|c|}{ 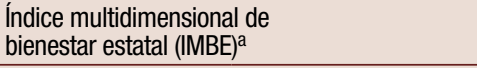 } & \multicolumn{2}{|c|}{$\begin{array}{l}\text { Índice de bienestar BLI, basado en el } \\
\text { Índice para una Vida Mejor de la OCDEb }\end{array}$} & \multicolumn{2}{|c|}{$\begin{array}{l}\text { Índice de bienestar SoL, basado en } \\
\text { Bérenger y Verdier-Chouchane }^{\mathrm{C}}\end{array}$} \\
\hline Dimensiones & Indicadores & Dimensiones & Indicadores & Dimensiones & Indicadores \\
\hline \multirow[t]{3}{*}{$\begin{array}{l}\text { Estándares de vida } \\
\text { material (ingreso, } \\
\text { consumo, riqueza) }\end{array}$} & $\begin{array}{l}\text { Mediana del ingreso } \\
\text { total corriente* }\end{array}$ & Vivienda & $\begin{array}{l}\text { Habitaciones por persona*; } \\
\text { Viviendas con techos de } \\
\text { materiales resistentes }\end{array}$ & \multirow[t]{5}{*}{$\begin{array}{l}\text { Bienestar } \\
\text { material }\end{array}$} & $\begin{array}{l}\text { Coeficiente de Gini de los } \\
\text { ingresos disponibles de } \\
\text { los hogares per cápita* }\end{array}$ \\
\hline & $\begin{array}{l}\text { Ingreso disponible } \\
\text { ajustado por el } \\
\text { coeficiente de Gini* }\end{array}$ & Ingreso & $\begin{array}{l}\text { Coeficiente de Gini de los ingresos } \\
\text { disponibles*; Ingreso equivalente } \\
\text { disponible*; población en } \\
\text { situación de pobreza*; población } \\
\text { en situación de pobreza extrema* }\end{array}$ & & $\begin{array}{l}\text { Mediana de ingresos } \\
\text { corrientes del hogar } \\
\text { per cápita* }\end{array}$ \\
\hline & $\begin{array}{l}\text { Mediana del gasto } \\
\text { total anual del hogar } \\
\text { ajustado por unidad de } \\
\text { consumo* }^{\star}\end{array}$ & Empleo & $\begin{array}{l}\text { Condiciones críticas de } \\
\text { ocupación }^{\star} \text {; informalidad laboral }{ }^{\star} \text {; } \\
\text { desempleo*; ocupación* }^{*}\end{array}$ & & $\begin{array}{l}\text { Ingreso disponible del } \\
\text { hogar per cápita* }\end{array}$ \\
\hline \multirow[t]{2}{*}{ Salud } & Situación de salud** & $\begin{array}{l}\text { Acceso a } \\
\text { servicios }\end{array}$ & $\begin{array}{l}\text { Acceso a servicios de salud*; } \\
\text { hogares con acceso a banda } \\
\text { ancha*; viviendas con acceso } \\
\text { a servicios básicos* }\end{array}$ & & $\begin{array}{l}\text { Gasto total anual del hogar } \\
\text { ajustado por unidad } \\
\text { de consumo* }\end{array}$ \\
\hline & Acceso a la salud* & Seguridad & $\begin{array}{l}\text { Homicidios*; confianza en } \\
\text { la policía**; percepción de } \\
\text { inseguridad }{ }^{\star \star} ; \text { incidencia } \\
\text { delictiva* }^{*}\end{array}$ & & $\begin{array}{l}\text { Ingresos corrientes del } \\
\text { hogar ajustados por el } \\
\text { coeficiente de Gini* }\end{array}$ \\
\hline Educación & $\begin{array}{l}\text { Escolaridad } \\
\text { acumulada* }\end{array}$ & Educación & $\begin{array}{l}\text { Niveles de educación*; deserción } \\
\text { escolar*; prueba Programa para la } \\
\text { Evaluación Internacional de } \\
\text { los Alumnos (PISA)* }\end{array}$ & \multirow[t]{2}{*}{ Educación } & \multirow[t]{2}{*}{ Escolaridad acumulada* } \\
\hline $\begin{array}{l}\text { Actividades } \\
\text { personales, incluido el } \\
\text { trabajo }\end{array}$ & $\begin{array}{l}\text { Satisfacción con la } \\
\text { actividad que realiza** }\end{array}$ & $\begin{array}{l}\text { Medio } \\
\text { ambiente }\end{array}$ & $\begin{array}{l}\text { Contaminación del aire*; } \\
\text { disposición de residuos* }\end{array}$ & & \\
\hline $\begin{array}{l}\text { Voz política y } \\
\text { gobernanza }\end{array}$ & Libertad de decisión** & $\begin{array}{l}\text { Compromiso } \\
\text { cívico y } \\
\text { gobernanza }\end{array}$ & $\begin{array}{l}\text { Participación cívica y política*; } \\
\text { participación electoral }^{\star} \text {; confianza } \\
\text { en la aplicación de la ley**; } \\
\text { percepción de ausencia } \\
\text { de corrupción** }\end{array}$ & \multirow[t]{3}{*}{ Salud } & Situación de salud actual $^{\star *}$ \\
\hline $\begin{array}{l}\text { Conexiones sociales } \\
\text { y relaciones }\end{array}$ & $\begin{array}{l}\text { Satisfacción con } \\
\text { la vida social**}\end{array}$ & Salud & $\begin{array}{l}\text { Esperanza de vida al nacer*; } \\
\text { salud autodeclarada**; obesidad*; } \\
\text { mortalidad materna*; mortalidad } \\
\text { infantil* }^{*}\end{array}$ & & $\begin{array}{l}\text { Satisfacción con la salud } \\
\text { actual }^{* *}\end{array}$ \\
\hline $\begin{array}{l}\text { Medio ambiente } \\
\text { (presente y futuro) }\end{array}$ & $\begin{array}{l}\text { Contaminación } \\
\text { del aire* }\end{array}$ & $\begin{array}{l}\text { Equilibrio } \\
\text { trabajo-vida }\end{array}$ & $\begin{array}{l}\text { Satisfacción con el tiempo } \\
\text { destinado al ocio**; empleados } \\
\text { que trabajan muchas horas* }\end{array}$ & & Acceso a la salud* \\
\hline \multirow[t]{2}{*}{$\begin{array}{l}\text { Inseguridad } \\
\text { (económica } \\
\text { y física) }\end{array}$} & $\begin{array}{l}\text { Satisfacción con la } \\
\text { seguridad ciudadana** }^{\star}\end{array}$ & $\begin{array}{l}\text { Comunidad } \\
\text { (conexiones } \\
\text { sociales) }\end{array}$ & Red social de apoyo** & $\begin{array}{l}\text { Bienestar } \\
\text { subjetivo }\end{array}$ & $\begin{array}{l}\text { Satisfacción de vida- } \\
\text { bienestar autodeclarado ** }\end{array}$ \\
\hline & & $\begin{array}{l}\text { Satisfacción } \\
\text { con la vida }\end{array}$ & Satisfacción con la vida** & & \\
\hline
\end{tabular}

Fuente: Elaboración propia, sobre la base de J. Stiglitz, A. Sen y J. Fitoussi, Report by the Commission on the Measurement of Economic Performance and Social Progress, París, The Commission, 2009 y V. Bérenger y A. Verdier-Chouchane, "Multidimensional measures of well-being: standard of living and quality of life across countries", World Development, vol. 35, N 7, Amsterdam, Elsevier, 2007.

Nota: $\quad$ * Indicador de bienestar objetivo; ${ }^{*}$ indicador de bienestar subjetivo.

a Basado en Stiglitz, Sen y Fitoussi (2009).

b Organización de Cooperación y Desarrollo Económicos (OCDE), 2015.

c Bérenger y Verdier-Chouchane, 2007.

\section{Datos}

Dada la disponibilidad de información a nivel microdato o estatal, la obtención de los datos representativos de cada entidad federativa se da de dos formas: i) los indicadores cuya información se obtiene directamente del estado, que no sufren ninguna transformación y se utilizan directamente, y ii) los indicadores obtenidos de información a nivel individual procedente de hogares encuestados, por 
lo que son transformados a partir de las medianas o los promedios estatales — según sea el casoponderados por el factor de ajuste (expansión) proporcionado por el INEGI. Cabe mencionar que este factor de expansión indica la forma en que el organismo nacional segmenta al total de población por estratos y unidades primarias de muestreo, permitiendo así hacer estimaciones de ella a partir de muestras representativas.

Los datos utilizados para el análisis proceden del INEGI y de la OCDE. Específicamente, los indicadores inghmed, educ y accesalud se han calculado a partir del Módulo de Condiciones Socioeconómicas de la Encuesta Nacional de Ingresos y Gastos de los Hogares (ENIGH) 2014; los indicadores situsalud, satisactiv, libertad, satissocial y satissecure, a partir del Módulo de Bienestar Autorreportado (BIARE) 2014; el indicador ingaj, de los datos del ingreso disponible de 2014 de la OCDE y del proyecto de Índice para una Vida Mejor del INEGI (datos del coeficiente de Gini); el indicador gpcmed se ha calculado a partir de la Encuesta Nacional de Gastos de los Hogares (ENGASTO) 2013, y el indicador $\mathrm{CO}_{2}$ de contaminación del aire también se ha tomado del proyecto de Índice para una Vida Mejor del INEGI.

Es importante señalar que el INEGI provee variables clave que permiten combinar la información proveniente de las bases de datos del Módulo de Condiciones Socioeconómicas y del Módulo de Bienestar Autorreportado de una misma persona u hogar, de tal forma que la información objetiva y subjetiva del bienestar puede analizarse conjuntamente. Asimismo, debe aclararse que, aunque la Encuesta Nacional de Ingresos y Gastos de los Hogares está disponible desde 1984, los datos del Módulo de Bienestar Autorreportado representativos a nivel estatal solo están disponibles desde 2014. Los demás datos estatales utilizados para construir el índice $B L I_{i}$ se encuentran en el proyecto de Índice para una Vida Mejor del INEGI. En la siguiente sección se muestran los resultados del análisis descrito, realizado por medio del software Stata 13.

\section{Resultados}

\section{Análisis de indicadores}

Una vez transformados los indicadores cuyos datos se obtuvieron a nivel individual, el análisis gráfico de las relaciones entre ellos aporta información interesante. En el gráfico 1 se muestra que, en general, existe una relación positiva bien definida entre los primeros seis indicadores (mediana del ingreso total corriente, ingreso disponible ajustado por el coeficiente de Gini, mediana del gasto en consumo, acceso y situación de salud y escolaridad). Como se recordará, los indicadores de ingreso, salud y educación, correspondientes a los seis indicadores mencionados, componen el índice de desarrollo humano. Todos los indicadores, excepto la situación de la salud actual e incluida la contaminación del aire, constituyen indicadores relacionados con el bienestar objetivo. El resto de los indicadores, relativos al bienestar subjetivo, en general no muestran una relación definida con los otros indicadores, sobre todo los del bienestar objetivo. Esto se puede deber a que los indicadores de bienestar subjetivo considerados abordan aspectos de vida que guardan poca relación con aspectos laborales o materiales. 
Gráfico 1

Relación entre los indicadores de bienestar

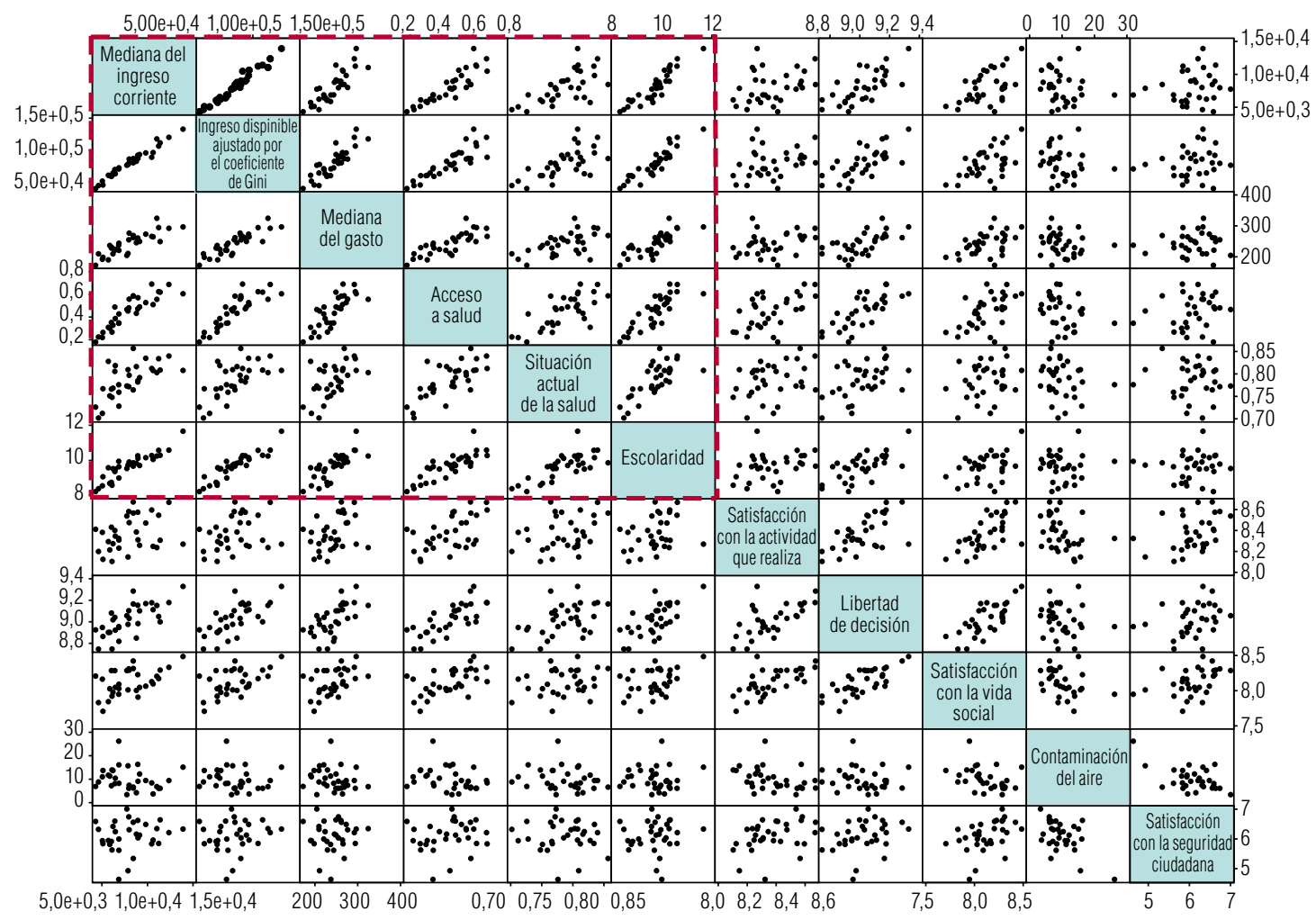

Fuente: Elaboración propia, sobre la base de datos del Instituto Nacional de Estadística y Geografía (INEGI) y de la Organización de Cooperación y Desarrollo Económicos (OCDE).

\section{Coeficiente alfa de Cronbach}

En la segunda etapa se realiza una prueba de confiabilidad mediante el alfa de Cronbach para verificar que los indicadores elegidos $x_{i j}$ aporten información a la escala de la variable subyacente "bienestar" (o $I M B E_{i}$ ). Para ello, es importante mencionar que el cálculo del coeficiente alfa de Cronbach se realiza con los datos estandarizados, dado que los indicadores se encuentran en unidades diferentes. En el cuadro 2 se muestra, en primer lugar, que la correlación media entre los 11 indicadores $x_{i j}$ es de 0,4855, un valor no muy alto, pero el alfa de Cronbach es de 0,9121. Aunque este valor es relativamente alto, es importante realizar pruebas de contribución para verificar que los indicadores considerados aporten de manera significativa y en la dirección esperada a la escala; de igual manera, es importante comprobar que el valor obtenido no esté comprometido por incumplir los supuestos de la prueba.

Cuadro 2

Alfa de Cronbach

\begin{tabular}{lc}
\hline Correlación media entre indicadores & 0,4855 \\
\hline Número de indicadores en la escala & 11 \\
\hline Coeficiente de confiabilidad de la escala & 0,9121
\end{tabular}

Fuente: Elaboración propia, sobre la base de datos del Instituto Nacional de Estadística y Geografía (INEGI). 
En el cuadro 3 se muestra información respecto a la contribución y correlación de cada indicador en la escala calculada por el alfa de Cronbach. La columna "Observaciones" indica que para el cálculo del alfa de Cronbach se obtuvo información de cada indicador procedente de los 32 estados del país, es decir, no se perdió ni omitió ningún dato. La columna "Signo" indica la dirección de cada indicador en la escala construida por el alfa de Cronbach, es decir, la forma en que cada uno de ellos contribuye a la escala de bienestar: el signo positivo muestra que un mayor valor del indicador correspondiente está relacionado con un mayor valor de la escala. Como se mencionó anteriormente, los indicadores muestran el signo esperado de acuerdo con la teoría: un mayor bienestar se ve reflejado en una mediana mayor del ingreso total del hogar corriente, un mayor ingreso disponible ajustado por el coeficiente de Gini, una mediana mayor del gasto en consumo, una mayor escolaridad alcanzada, un mayor acceso a la salud y una mejor situación de esta, una mayor satisfacción con la actividad realizada, una mayor libertad en la toma de decisiones, una mayor satisfacción con la vida social, una menor contaminación del aire y una mayor satisfacción con la seguridad ciudadana.

Cuadro 3

Detalles de correlaciones del alfa de Cronbach

\begin{tabular}{|c|c|c|c|c|c|c|}
\hline Indicador & Observaciones & Signo & ITC ${ }^{\mathrm{a}}$ & $\mathrm{IRC}^{\mathrm{b}}$ & $\| C^{c}$ & $\mathrm{C}$-a cont ${ }^{\mathrm{d}}$ \\
\hline $\begin{array}{l}\text { Mediana de ingresos corrientes } \\
\text { del hogar per cápita }\end{array}$ & 32 & + & 0,8870 & 0,8554 & 0,4574 & 0,8940 \\
\hline $\begin{array}{l}\text { Ingresos corrientes del hogar } \\
\text { ajustados por Gini }\end{array}$ & 32 & + & 0,8867 & 0,8550 & 0,4575 & 0,8940 \\
\hline $\begin{array}{l}\text { Mediana del gasto total anual } \\
\text { por persona ajustado }\end{array}$ & 32 & + & 0,8125 & 0,7629 & 0,4707 & 0,8989 \\
\hline Acceso a salud & 32 & + & 0,9207 & 0,8979 & 0,4514 & 0,8916 \\
\hline Situación de salud actual & 32 & + & 0,6801 & 0,6040 & 0,4943 & 0,9072 \\
\hline Escolaridad & 32 & + & 0,8230 & 0,7758 & 0,4688 & 0,8982 \\
\hline $\begin{array}{l}\text { Satisfacción con la actividad } \\
\text { que realiza }\end{array}$ & 32 & + & 0,6354 & 0,5517 & 0,5023 & 0,9098 \\
\hline Libertad de decisión & 32 & + & 0,8367 & 0,7927 & 0,4664 & 0,8973 \\
\hline Satisfacción con la vida social & 32 & + & 0,7361 & 0,6704 & 0,4843 & 0,9038 \\
\hline Contaminación del aire & 32 & - & 0,4234 & 0,3132 & 0,5401 & 0,9215 \\
\hline $\begin{array}{l}\text { Satisfacción con la seguridad } \\
\text { ciudadana }\end{array}$ & 32 & + & 0,3835 & 0,2699 & 0,5472 & 0,9236 \\
\hline Escala & & & & & 0,4855 & 0,9121 \\
\hline
\end{tabular}

Fuente: Elaboración propia, sobre la base de datos del Instituto Nacional de Estadística y Geografía (INEGl) y la Organización de Cooperación y Desarrollo Económicos (OCDE).

a Correlación indicador-escala.

b Correlación indicador-resto.

c Correlación entre los indicadores.

d Contribución al alfa de Cronbach si se omite el indicador.

En la columna "ITC" del cuadro 3 se indica la correlación de cada indicador con la escala obtenida por el alfa de Cronbach de los 11 indicadores incluidos; en este caso los que menor correlación tienen son la satisfacción con la seguridad ciudadana y la contaminación del aire. Dado que la inclusión de estos indicadores influye en la escala obtenida, es conveniente apoyarse en la información de la columna "IRC" (StataCorp, 2013), en la que se indica la correlación de cada indicador con la escala obtenida a partir de los otros diez restantes; en este caso la correlación de la satisfacción con la seguridad ciudadana y la contaminación del aire es todavía menor, lo que sugiere que estos indicadores se ajustan muy poco a la escala general obtenida.

En la columna "IIC" del cuadro 3 se muestra el promedio de las correlaciones entre los indicadores excepto el de la fila indicada; en este caso, como se mencionó anteriormente, se observa que la correlación media entre los indicadores es de 0,4855, pero si se omite el indicador de satisfacción con 
la seguridad ciudadana o la contaminación del aire, la correlación media aumenta a 0,5472 o 0,5401, respectivamente. Finalmente, en la columna "C-a cont" se brinda información sobre el cambio del alfa de Cronbach al omitir un indicador; nuevamente se observa que la omisión de alguno de los dos indicadores mencionados mejora la escala obtenida, pues el alfa aumenta a 0,9236 o 0,9215, respectivamente.

Un aspecto importante que debe considerarse en cuanto a estos resultados es que, a pesar de que el alfa de Cronbach obtenido es relativamente alto, el número de observaciones considerado en el cálculo es relativamente bajo y, por tanto, de acuerdo con la literatura, la confiabilidad del análisis estadístico puede estar comprometida. Específicamente, Yurdugül (2008) argumenta que la confiabilidad de la prueba, además de depender del número de observaciones, depende del autovalor (eigenvalue) obtenido mediante el análisis de componentes principales; según sus pruebas mediante el método de Montecarlo, una muestra de 30 observaciones es confiable si el autovalor del primer componente obtenido en el análisis de componentes principales es mayor que seis. Si se realiza dicho análisis con los datos de este estudio, se observa que el autovalor del primer componente es mayor que seis (véase el cuadro 5), por lo que puede argumentarse que el alfa de Cronbach obtenido es confiable.

Los resultados obtenidos hasta aquí presentan algunos aspectos que pueden discutirse para proceder con confianza al análisis de factores: aunque los indicadores de satisfacción con la seguridad ciudadana y la contaminación del aire no aportan una gran cantidad de información a la escala obtenida por el coeficiente alfa, su omisión no eleva la confiabilidad de la escala significativamente; en el mismo sentido, los indicadores de estándares de vida material, educación y salud pueden estar aportando duplicidad de información. Sin embargo, todos estos indicadores $x_{i j}$, además de ser importantes en la teoría de Stiglitz, Sen y Fitoussi (2009), no solo no comprometen la validez del análisis estadístico, sino que también aportan información respecto de la multidimensionalidad del bienestar, por lo que no se han omitido del análisis posterior.

\section{Análisis de factores de componentes principales}

Como se mencionó anteriormente, la OCDE (2008) enfatiza la necesidad de que los indicadores de interés $x_{i j}$ estén correlacionados entre sí; de otro modo no tiene sentido realizar un análisis de factores de componentes principales. En cuanto a la tercera etapa del análisis, a partir de la matriz de correlaciones que se presenta en el cuadro 4 se puede confirmar la existencia de dos grupos principales de indicadores: por una parte, los estándares de vida material y los indicadores de las dimensiones de educación y salud presentan correlaciones altas y significativas entre sí, y bajas o no significativas con los demás indicadores; por otra parte, existen correlaciones significativas (aunque no tan altas) entre el resto de los indicadores, relacionados con la satisfacción del individuo, lo que refuerza la decisión de no omitirlos del análisis. Otro aspecto importante es que el signo de las correlaciones es el esperado conforme a la teoría y a lo discutido anteriormente en el análisis del alfa de Cronbach. Así, el conjunto de indicadores $x_{i j}$ tiene el comportamiento deseado y se espera que no presente problemas en el análisis de factores.

En cuanto a la cuarta etapa, en el cuadro 5 se presenta el análisis de factores de componentes principales de los indicadores $x_{i j}$ seleccionados. La parte superior de los resultados muestra que para el cálculo se utilizó información de los 11 indicadores para las 32 entidades federativas consideradas. Es evidente que, de acuerdo con los criterios expuestos en la metodología, solamente los dos primeros factores deberán ser utilizados para la construcción del índice de bienestar mostrado en la ecuación (1), pues son los únicos que tienen un autovalor mayor que 1 (columna 2) y explican más del $60 \%$ de la varianza total (columnas 4 y 5). A fin de confirmar la presencia de correlación entre los indicadores empleados, se realiza una prueba de independencia entre los indicadores; es decir, la hipótesis nula es que los elementos situados debajo de la diagonal de la matriz de varianza-covarianza tienen un valor de 0 , y es rechazada al 95\%, como se muestra en la parte inferior del cuadro. 
Cuadro 4

Matriz de correlaciones entre los indicadores de bienestar a nivel estatal

\begin{tabular}{|c|c|c|c|c|c|c|c|c|c|c|}
\hline & inghmed & ingaj & gpcmed & accesalud & situsalud & educ & satisactiv & libertad & satissocial & $\mathrm{CO}_{2}$ \\
\hline inghmed & 1 & & & & & & & & & \\
\hline ingaj & $0,9806^{*}$ & 1 & & & & & & & & \\
\hline gpcmed & $0,8467^{\star}$ & $0,8709^{\star}$ & 1 & & & & & & & \\
\hline accesalud & $0,8819^{*}$ & $0,8718^{\star}$ & $0,8000^{*}$ & 1 & & & & & & \\
\hline situsalud & $0,6512^{*}$ & $0,6549^{*}$ & $0,5576^{\star}$ & $0,7082^{\star}$ & 1 & & & & & \\
\hline educ & $0,9091^{*}$ & $0,9103^{*}$ & $0,7684^{*}$ & $0,8274^{\star}$ & $0,7321^{*}$ & 1 & & & & \\
\hline satisactiv & 0,3163 & 0,2969 & 0,3348 & $0,5404^{\star}$ & 0,3204 & 0,3107 & 1 & & & \\
\hline libertad & $0,7030^{*}$ & $0,6991^{*}$ & $0,6328^{*}$ & $0,7464^{*}$ & $0,4850^{*}$ & $0,6753^{*}$ & $0,6096^{*}$ & 1 & & \\
\hline satissocial & $0,5085^{\star}$ & $0,5371^{*}$ & $0,4715^{\star}$ & $0,5633^{*}$ & 0,2431 & $0,4146^{*}$ & $0,6493^{*}$ & $0,7337^{\star}$ & 1 & \\
\hline co2 & $-0,1810$ & $-0,1440$ & $-0,1839$ & $-0,2881$ & $-0,1874$ & 0 & $-0,3584^{*}$ & $-0,1461$ & $-0,3723^{*}$ & 1 \\
\hline satissecure & 0,1402 & 0,1502 & 0,0542 & 0,1610 & $-0,0818$ & 0,0571 & $0,3623^{*}$ & 0,2837 & $0,4142^{*}$ & $-0,5363^{*}$ \\
\hline
\end{tabular}

Fuente: Elaboración propia, sobre la base de datos del Instituto Nacional de Estadística y Geografía (INEGl) y de la Organización de Cooperación y Desarrollo Económicos (OCDE).

Nota: Los indicadores son: mediana del ingreso total corriente (inghmed); ingreso disponible ajustado por el coeficiente de Gini estatal (ingaj); mediana del gasto total anual del hogar ajustado por unidad de consumo (gpcmed); media del acceso a la salud (accesalud); media de la situación de salud declarada (situsalud); media de la escolaridad acumulada (educ); media de la satisfacción con la actividad que se realiza (satisactiv); media de la libertad de decisión declarada (libertad); media de la satisfacción con la vida social (satissocial); contaminación del aire $\left(\mathrm{CO}_{2}\right)$, y media de la satisfacción con la seguridad ciudadana (satissecure).

Significativo al 95\%.

Cuadro 5

Análisis de factores de componentes principales

\begin{tabular}{|c|c|c|c|c|}
\hline \multirow{2}{*}{\multicolumn{2}{|c|}{$\begin{array}{l}\text { Análisis de factores/correlación } \\
\text { Método: factores de componentes principales }\end{array}$}} & \multicolumn{3}{|c|}{ Número de observaciones = 32} \\
\hline & & & s retenidos $=$ & \\
\hline \multicolumn{2}{|c|}{ Rotación: (sin rotación) } & \multicolumn{3}{|c|}{ Número de parámetros = 21} \\
\hline Factor & Autovalor & Diferencia & Proporción & Acumulado \\
\hline Factor 1 & 6,31464 & 4,3883 & 0,5741 & 0,5741 \\
\hline Factor 2 & 1,92634 & 1,07485 & 0,1751 & 0,7492 \\
\hline Factor 3 & 0,85149 & 0,16178 & 0,0774 & 0,8266 \\
\hline Factor 4 & 0,6897 & 0,25333 & 0,0627 & 0,8893 \\
\hline Factor 5 & 0,43638 & 0,14494 & 0,0397 & 0,929 \\
\hline Factor 6 & 0,29143 & 0,10878 & 0,0265 & 0,9555 \\
\hline Factor 7 & 0,18265 & 0,03209 & 0,0166 & 0,9721 \\
\hline Factor 8 & 0,15056 & 0,0605 & 0,0137 & 0,9857 \\
\hline Factor 9 & 0,09006 & 0,03367 & 0,0082 & 0,9939 \\
\hline Factor 10 & 0,05639 & 0,04603 & 0,0051 & 0,9991 \\
\hline Factor 11 & 0,01036 & - & 0,0009 & 1,0000 \\
\hline
\end{tabular}

Prueba de cociente de probabilidad: independiente/agrupado: chi2(55) =372,41 Prob>chi2 =0,0000.

Fuente: Elaboración propia, sobre la base de datos del Instituto Nacional de Estadística y Geografía (INEGl) y de la Organización de Cooperación y Desarrollo Económicos (OCDE).

Como se mencionó en la sección anterior, se realiza la rotación de los factores mediante el método ortogonal varimax, para obtener posteriormente los ponderadores o cargas de factores al cuadrado normalizados $w_{j k}$. En el cuadro 6 se muestran los resultados obtenidos con la rotación. La parte superior del cuadro es similar a la del cuadro 5 , salvo que ahora se indica que los factores se 
sometieron a rotación por el método ortogonal varimax. En la segunda parte del cuadro se muestra la varianza de cada factor (autovalor) y su proporción respecto a la varianza de ambos ( $W_{k}$ en la ecuación (1)); es claro que el primer factor es el que tiene mayor ponderación (casi un 70\%). En la tercera parte se presentan diversas columnas: la segunda y la tercera columnas muestran las cargas correspondientes a los dos factores ya transformadas por la rotación. Se puede observar que la magnitud de las cargas separa a los indicadores en cada factor de acuerdo con lo observado en la matriz de correlaciones y los diagramas de dispersión: los indicadores de estándares de vida material, salud y educación tienen cargas mayores en el primer factor, y el resto de los indicadores (excepto libertad de decisión) las tienen en el segundo factor. La columna referente a unicidad indica que estos factores no están dejando fuera una parte considerable de la varianza de ningún indicador; de acuerdo con StataCorp (2013), un valor superior a 0,6 se considera alto. En este sentido, el indicador de contaminación del aire es el que está perdiendo más información (aproximadamente un 46\% de su varianza) en el análisis y en la posterior construcción del índice.

Cuadro 6

Rotación varimax y obtención de ponderadores

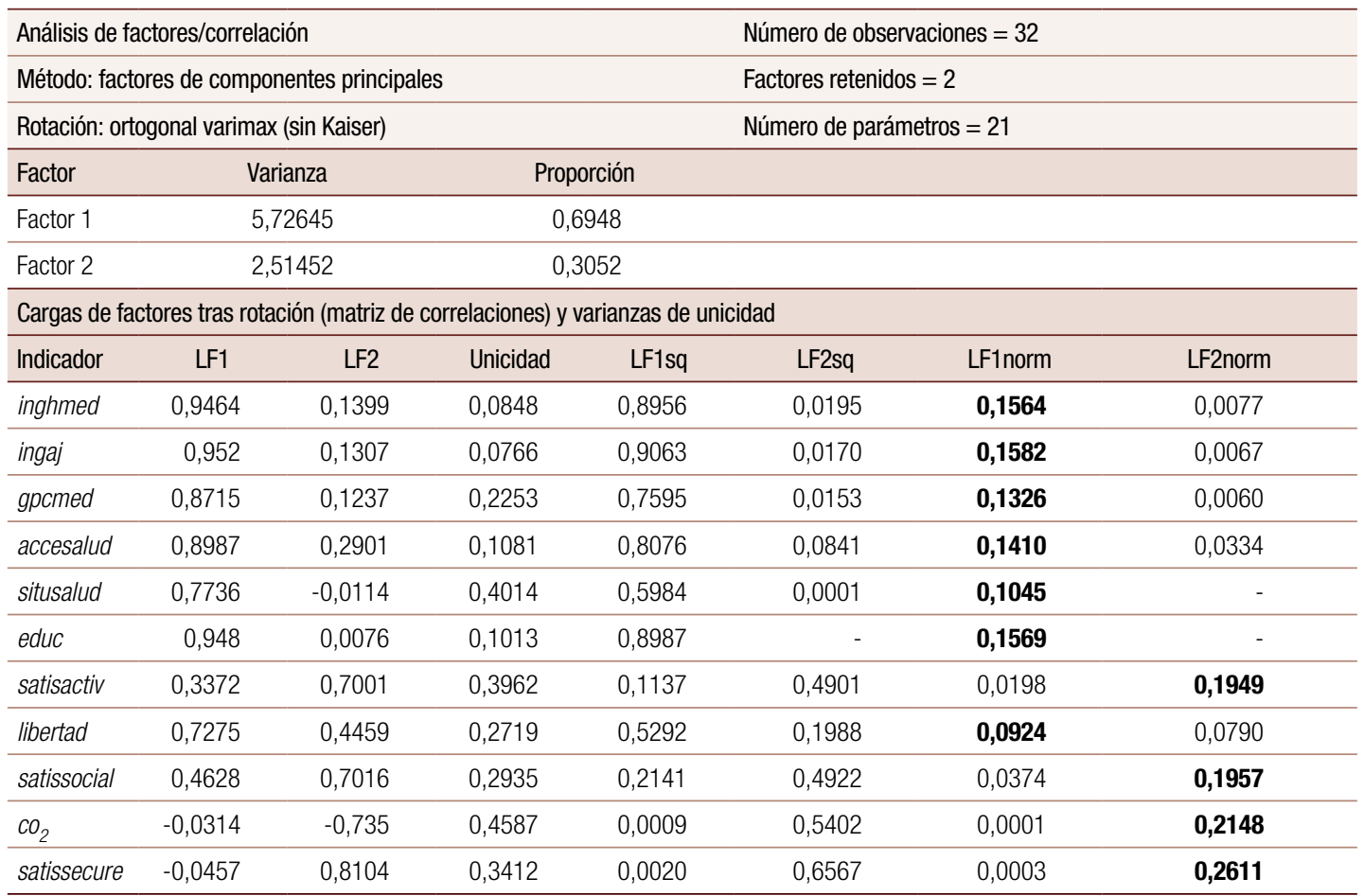

Fuente: Elaboración propia, sobre la base de datos del Instituto Nacional de Estadística y Geografía (INEGl) y de la Organización de Cooperación y Desarrollo Económicos (OCDE).

Nota: $L^{*} F^{\star}=$ carga del factor $1 \circ 2$; unicidad = porcentaje de varianza que no explican los factores; $L F^{\star} s q=$ carga al cuadrado; $\mathrm{LF}^{\star}$ norm = carga al cuadrado normalizada por la varianza del factor. Los indicadores son: mediana del ingreso total corriente (inghmed); ingreso disponible ajustado por el coeficiente de Gini estatal (ingaj); mediana del gasto total anual del hogar ajustado por unidad de consumo (gpcmed); media del acceso a la salud (accesalud); media de la situación de salud declarada (situsa/ud); media de la escolaridad acumulada (educ); media de la satisfacción con la actividad que se realiza (satisactiv); media de la libertad de decisión declarada (libertad); media de la satisfacción con la vida social (satissocial); contaminación del aire $\left(\mathrm{CO}_{2}\right)$, y media de la satisfacción con la seguridad ciudadana (satissecure).

En las columnas quinta y sexta del cuadro 6 se muestran las cargas al cuadrado, y en las últimas dos columnas se muestran las cargas normalizadas, que servirán de ponderadores para construir el $I M B E_{i}$ que se presenta en la ecuación (1). De acuerdo con OCDE (2008), es evidente que la rotación varimax permite obtener ponderaciones de cada indicador $x_{i j}$ que sean significativas en un 
solo factor; dichos ponderadores ( $w_{j k}$ en la ecuación (1)) se muestran en letras negritas, lo que indica que los indicadores relativos a los estándares de vida material, educación, salud y libertad de decisión se deben incluir en el primer factor y el resto de los indicadores en el segundo.

Antes de construir el índice de bienestar, de acuerdo con lo mencionado en la sección sobre metodología, se calcula la medida de Kaiser-Meyer-Olkin (KMO) de la comunalidad de los indicadores para sustentar la validez de un análisis de factores. De acuerdo con la escala provista por Kaiser (1974), citado en StataCorp (2013), los valores entre 0,80 y 0,89 son "meritorios" y los valores entre 0,70 y 0,79 son "regulares" pero aceptables (OCDE, 2008). Los resultados que se presentan en el cuadro 7 indican que la medida del factor utilizado es aceptable, a pesar de que los dos últimos indicadores tienen un valor bastante bajo.

Cuadro 7

Medida de Kaiser-Meyer-Olkin

\begin{tabular}{lc}
\hline Indicador & Medida de Kaiser-Meyer-Olkin (KM0) \\
\hline inghmed & 0,7370 \\
\hline ingaj & 0,6843 \\
\hline gpcmed & 0,7913 \\
\hline accesalud & 0,919 \\
\hline situsalud & 0,6634 \\
\hline educ & 0,8887 \\
\hline satisactiv & 0,6785 \\
\hline libertad & 0,8087 \\
\hline satissocial & 0,6356 \\
\hline co ${ }_{2}$ & 0,2909 \\
\hline satissecure & 0,3355 \\
\hline Total & 0,7139 \\
\hline
\end{tabular}

Fuente: Elaboración propia.

Nota: Los indicadores son: mediana del ingreso total corriente (inghmed); ingreso disponible ajustado por el coeficiente de Gini estatal (ingaj); mediana del gasto total anual del hogar ajustado por unidad de consumo (gpcmed); media del acceso a la salud (accesalud); media de la situación de salud declarada (situsalud); media de la escolaridad acumulada (educ); media de la satisfacción con la actividad que se realiza (satisactiv); media de la libertad de decisión declarada (libertad); media de la satisfacción con la vida social (satissocial); contaminación del aire $\left(\mathrm{CO}_{2}\right)$, y media de la satisfacción con la seguridad ciudadana (satissecure).

Finalmente, se construye el índice de bienestar para los 32 estados, sobre la base de la ecuación (1) y los ponderadores $W_{k}$ y $w_{j k}$ obtenidos a partir del cuadro 6. De acuerdo con Aiginger y Firgo (2015), se registra el valor del índice normalizado mediante el método min-max para que el estado con mayor valor de bienestar tenga $1 \mathrm{y}$ el estado con menor valor tenga 0 . El $I M B E_{i}$ de las entidades federativas de México se presenta en el gráfico 2, donde el eje $y$ indica el valor normalizado del $I M B E_{i}$ y en el eje $x$ se ordenan las 32 entidades federativas del país de mayor a menor nivel de bienestar.

En general, se puede observar que el $I M B E_{i}$ refleja la ponderación relativamente alta que obtuvieron los indicadores de estándares de vida material en el primer componente obtenido mediante el análisis de factores principales y, por tanto, en el índice. Las entidades federativas con mayor bienestar son Ciudad de México y Nuevo León, en tanto que las entidades federativas con menor bienestar son Puebla y Oaxaca. Además, es interesante observar que las brechas de bienestar agrupan a las entidades federativas en tres sectores: por una parte, se encuentra el grupo de entidades 
federativas con un nivel de bienestar bajo (menor que 0,4), que incluye desde Oaxaca hasta San Luis Potosí; en segundo lugar, está el grupo de las entidades federativas con un nivel de bienestar medio (entre 0,4 y 0,6), que incluye desde Durango hasta Yucatán, y posteriormente, se encuentra el grupo de entidades federativas con un nivel de bienestar alto (mayor que 0,6), que incluye desde Nayarit hasta Ciudad de México.

\section{Gráfico 2}

México: índice multidimensional de bienestar estatal (IMBE), 2014

(En valores normalizados entre 0 y 1 )

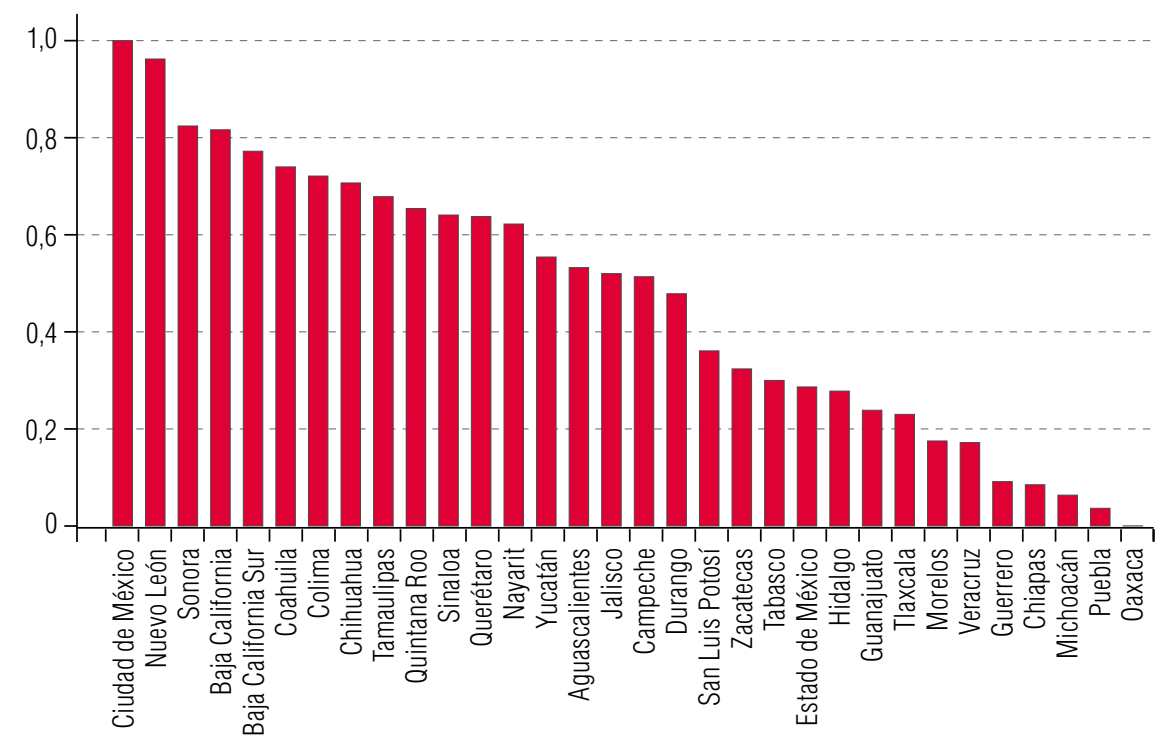

Fuente: Elaboración propia, sobre la base de datos del Instituto Nacional de Estadística y Geografía (INEGl) y de la Organización de Cooperación y Desarrollo Económicos (OCDE).

A continuación, como quinta etapa del análisis descrito en la sección anterior, es interesante indagar si estos resultados son similares a la información aportada por otros indicadores relativos al bienestar. Por ello se realiza una comparación en primer lugar con indicadores ya construidos (IDH e indicador de satisfacción de vida en general) y posteriormente con los índices $S o L_{i}$ y $B L I_{i}$. En el gráfico 3 se presenta la comparación de los resultados del $I M B E_{i}$ en primer término con el IDH estatal de 2012 (el más reciente provisto por el Programa de las Naciones Unidas para el Desarrollo en México), que agrupa información de ingresos, educación y salud, y en segundo término con el indicador de satisfacción de vida provisto por el Módulo de Bienestar Autorreportado (BIARE) de 2014.

En primer lugar, el coeficiente de correlación de Pearson presentado en el gráfico 3 señala que ambos indicadores tienen una relación positiva con el $I M B E_{i}$, es decir, que una variación positiva en un indicador está relacionada con una variación positiva en el otro, y es mucho mayor en el caso del índice de desarrollo humano. Por otra parte, el coeficiente de correlación tau de Kendall indica que la relación de rango o clasificación entre los indicadores no es tan alta, es decir, la variación en el bienestar de las entidades federativas no es regular para los dos indicadores considerados en cada caso, de manera que las posiciones que ocupa cada estado difieren relativamente entre indicadores, sobre todo en la comparación con el indicador referente al bienestar autorreportado. Hasta ahora puede argumentarse que estos resultados confirman la importancia que tiene el ingreso en el bienestar total de la población mexicana, y parecen respaldar lo que se ha encontrado en diversos estudios respecto a la baja importancia del ingreso en el bienestar subjetivo de las personas. 


\section{Gráfico 3}

México: relación del índice multidimensional de bienestar estatal (IMBE) con el índice de desarrollo humano (IDH) de 2012 y el indicador de satisfacción de vida

del Módulo de Bienestar Autorreportado (BIARE) de 2014

A. Relación con el IDH

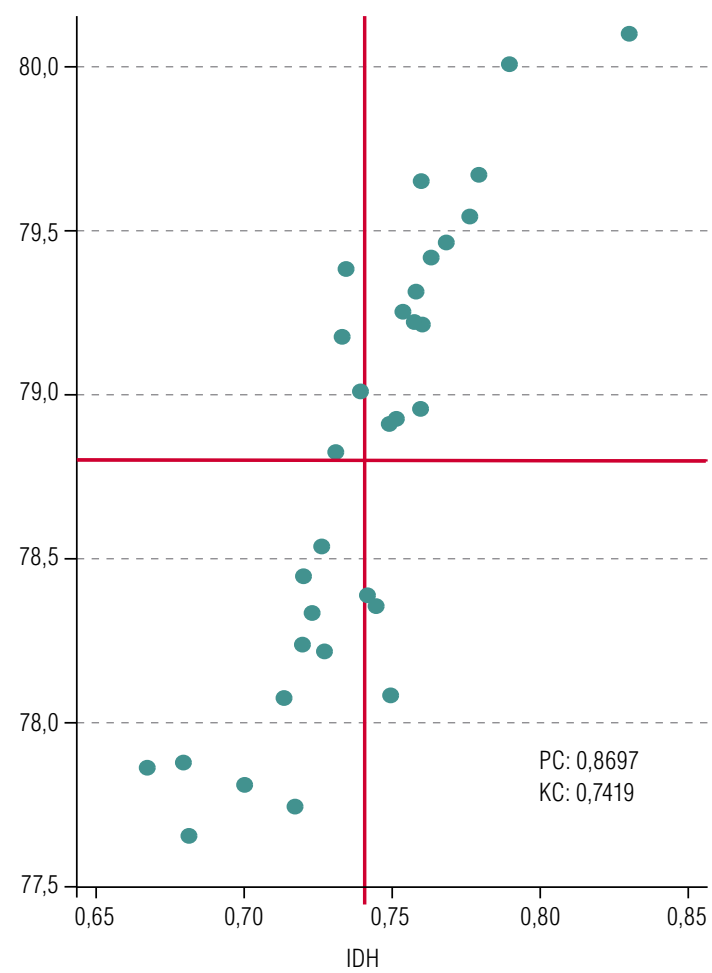

B. Relación con el indicador de satisfacción de vida del Módulo BIARE

- Media del indicador

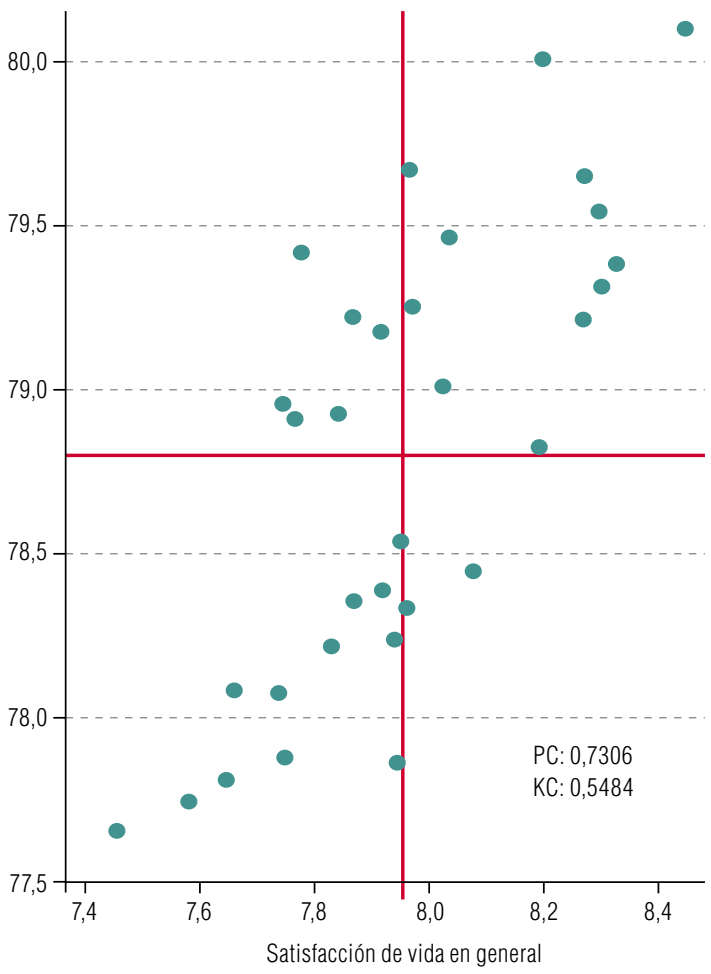

Fuente: Elaboración propia, sobre la base de datos del Instituto Nacional de Estadística y Geografía (INEGl), el Programa de las Naciones Unidas para el Desarrollo (PNUD) y la Organización de Cooperación y Desarrollo Económicos (OCDE).

Nota: PC: correlación de Pearson; KC: correlación tau de Kendall.

En cuanto a los indicadores adaptados de otras propuestas, se reitera que se consideró la propuesta de Bérenger y Verdier-Chouchane (2007) (véase el cuadro 1), agregando la dimensión de bienestar autorreportado para realizar una comparación más adecuada con el $I M B E_{i}^{5}$; como se mencionó, a este índice se le denomina $S o L_{i}$ por basarse en las dimensiones del indicador sobre estándares de vida (standards of living) de estas autoras. Asimismo se retomaron las dimensiones, los indicadores ${ }^{6}$ y el proceso ${ }^{7}$ empleados para la construcción del índice de bienestar global de la OCDE (2015), pero en vez de utilizar los índices de Mazziotta-Pareto ajustados (AMPI) para la construcción

5 Al hacer las pruebas de confiabilidad se encontró que el indicador del coeficiente de Gini del ingreso disponible, además de no contribuir a la escala subyacente del alfa de Cronbach, no tenía una correlación significativa con ningún otro indicador, por lo que no se consideró en la construcción del índice; al respecto, se hicieron pruebas para comprobar la confiabilidad y el cumplimiento de los supuestos del análisis de factores al omitir dicho indicador, y no encontró problema alguno.

6 Se consideran los indicadores más recientes disponibles en el Índice para una Vida Mejor del INEGI. Se enfatiza que los indicadores tomados del Módulo de Bienestar Autorreportado, a diferencia de los empleados por la OCDE (2015), son representativos por entidad federativa. Para la construcción de las dimensiones, los indicadores cuya relación con el bienestar era negativa (por ejemplo, el indicador pobreza) fueron transformados (mediante la inversa) de manera que todos tuvieran una relación positiva con el bienestar.

7 Se refiere a las dos etapas de la construcción del índice general de bienestar. En la primera etapa se construyen las dimensiones a partir de los indicadores correspondientes, y en la segunda se construye el índice a partir de las dimensiones obtenidas. En ambos casos se utilizó el análisis de factores. 
de las dimensiones ${ }^{8}$ y el índice general de bienestar, se utilizó la misma metodología empleada para obtener el $I M B E_{i}$, es decir, se aplicó el análisis de factores para obtener estadísticamente diferentes ponderaciones por dimensión e indicador; a este índice se le denominó $B L I_{i}$, por referirse a los datos relativos al Índice para una Vida Mejor (Better Life Index). Cabe mencionar que, para contar con una comparación más completa, se muestra también el índice informado para 2014 por la OCDE (2015), al que se hace referencia simplemente como indicador AMPI.

En el proceso de cálculo de los índices, es importante notar que en relación con el índice $S_{o} L_{i}$ los indicadores obtuvieron una ponderación similar (aproximadamente del 10\%) mediante el método de análisis de factores; las menores fueron las correspondientes a satisfacción de vida (casi un 8\%) y situación de salud $(9,3 \%)$, en tanto que la mayor fue la correspondiente a ingreso disponible, con un $13 \%$. Por su parte, las dimensiones con mayor ponderación en la construcción del índice $B L I_{i}$ fueron medio ambiente (9\%) y salud (8,84\%); las de menor ponderación fueron, aparte de participación cívica y política $(2,42 \%)$, las relativas al bienestar subjetivo: satisfacción con la vida $(3,73 \%)$, equilibrio entre trabajo y vida $(4,78 \%)$ y conexiones sociales $(5,12 \%)$. Una vez obtenidos los ponderadores de cada dimensión, se construyeron los índices respectivos, que se presentan en el cuadro 8.

En el cuadro 8 se muestra el valor de cada indicador de bienestar, así como la posición de cada entidad federativa en cada uno de ellos. Todos los indicadores de bienestar, excepto el de satisfacción con la vida (BIARE), sitúan a Baja California Sur, Nuevo León y Sonora entre las cinco entidades federativas con mayor bienestar, y a Chiapas, Guerrero, Oaxaca y Puebla entre las que presentan menor bienestar. Por su parte, los indicadores $I M B E_{i}$, So $L_{i}$ e IDH ubican a los estados prácticamente en la misma posición de bienestar, clasificando entre las mejores posiciones a Ciudad de México y Nuevo León, y entre las peores a Chiapas y Oaxaca nuevamente.

El gráfico 4 brinda una mejor apreciación de la similitud y las diferencias entre los índices adaptados y el indicador que se ha denominado AMPI. Es evidente que la mayor correlación se da entre el $I M B E_{i}$ y el índice $S o L_{i}$, lo que no es sorprendente, puesto que el $I M B E_{i}$ solo se diferencia del índice $S o L_{i}$ por incluir más indicadores referentes al bienestar subjetivo, los que tuvieron una ponderación relativamente menor en la construcción del índice. Sin embargo, la correlación entre el $I M B E_{i}$ y el índice $B L I_{i}$ es más interesante, dadas las diferencias importantes en la construcción de ambos; a pesar de que incluyen distinto número de dimensiones y consideran indicadores diferentes en número y características (salvo las características objetivas o subjetivas del bienestar), la correlación entre ambos es muy alta. Incluso al considerar la correlación tau de Kendall se observa que ambos índices ubican a las distintas entidades federativas prácticamente en la misma posición de bienestar.

\footnotetext{
8 En el caso de los estados de Michoacán, Oaxaca y Sonora, la dimensión de educación se construyó sin utilizar la información del Programa para la Evaluación Internacional de los Alumnos (PISA), por no estar disponible.
} 


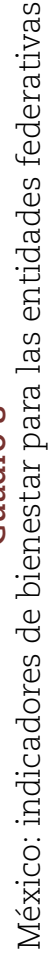

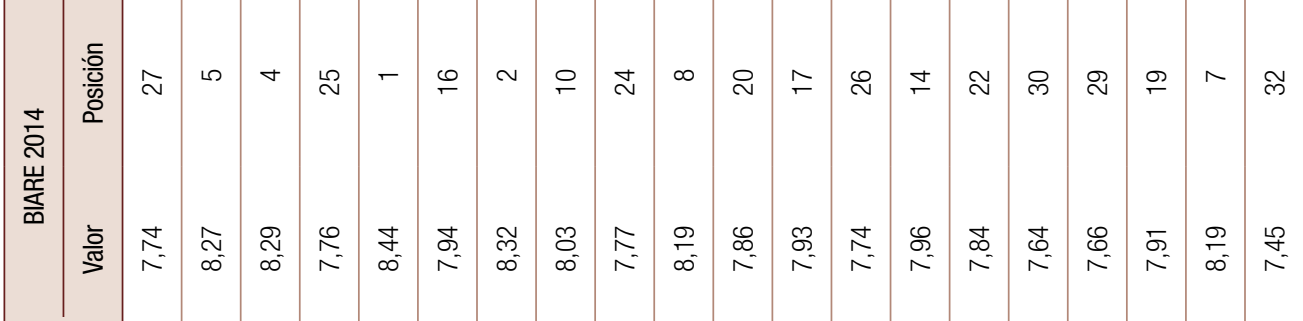
:

혼

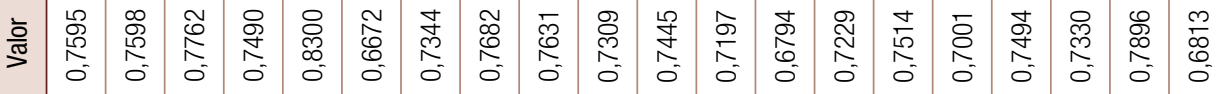

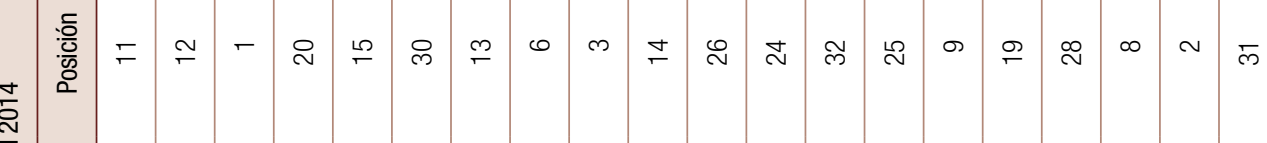

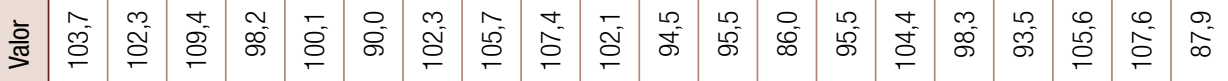

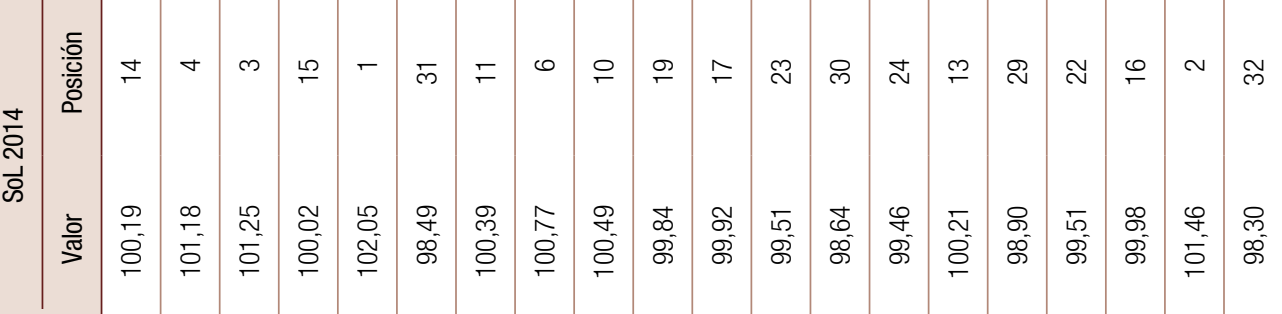

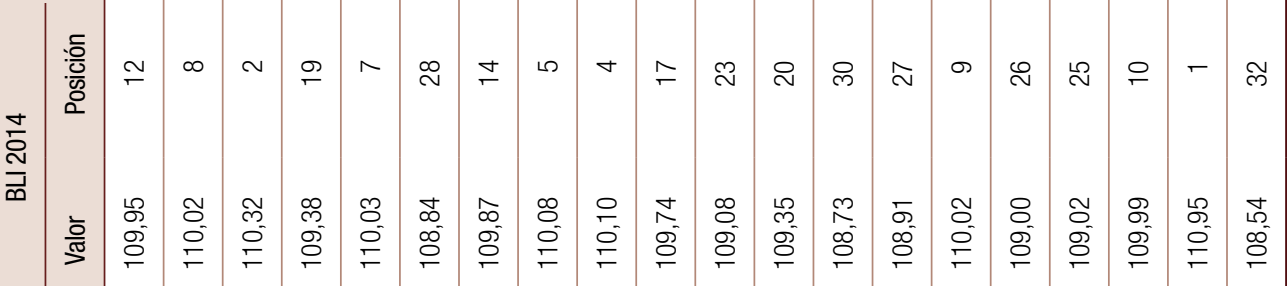

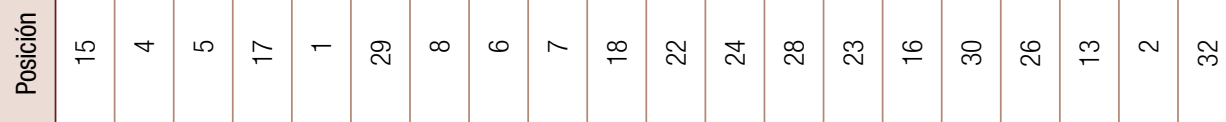

岕

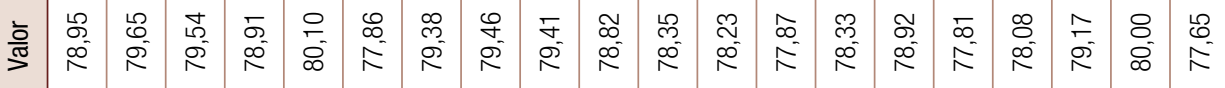

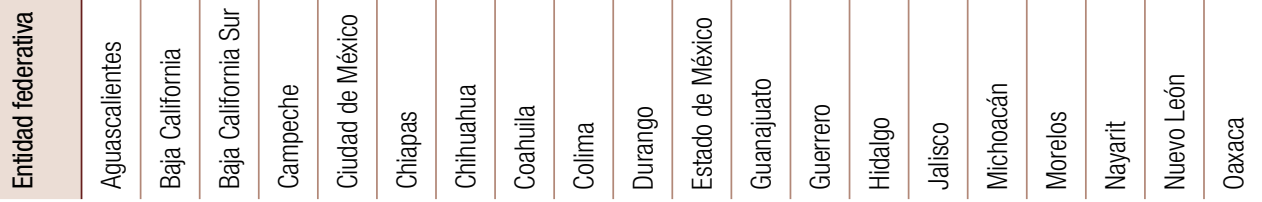




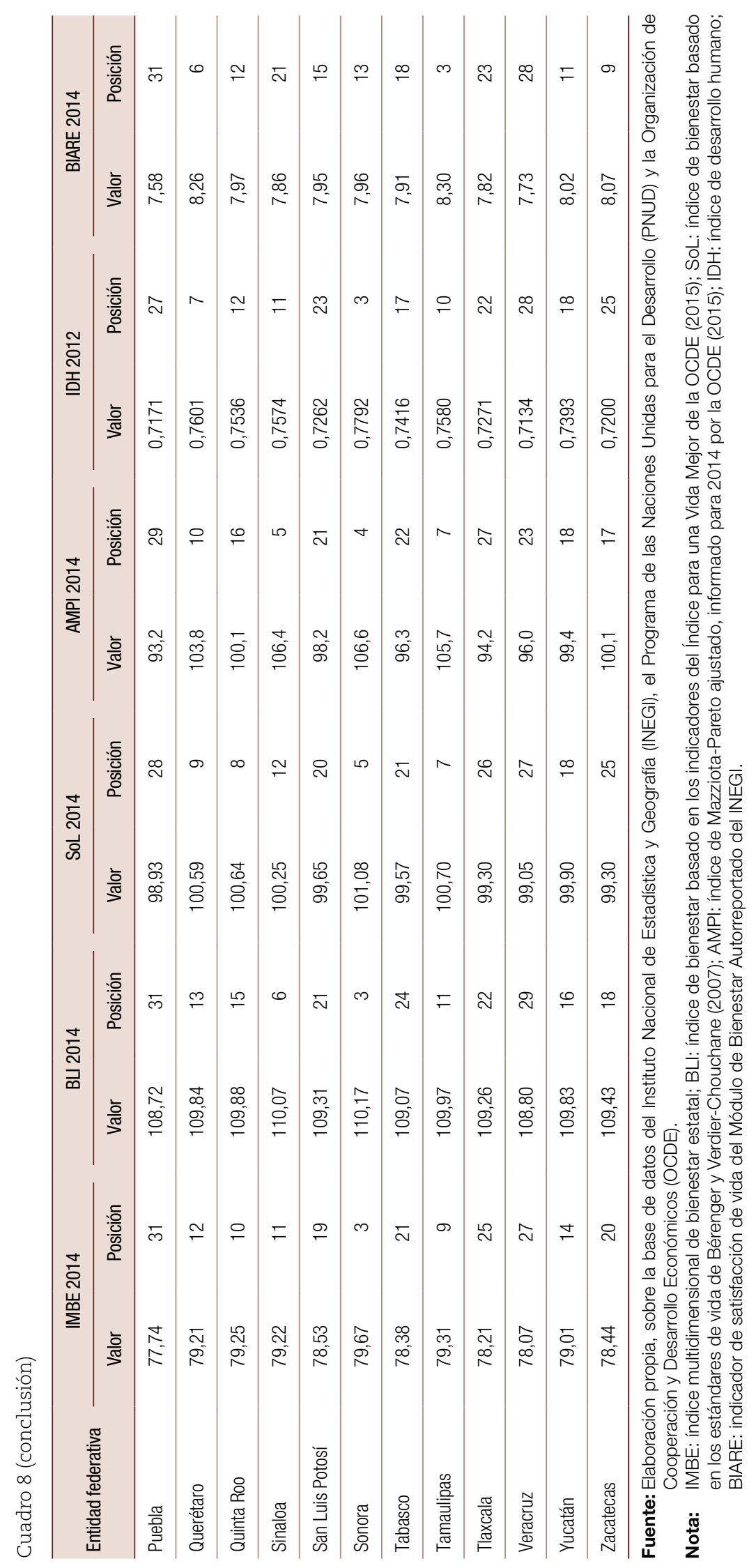


Gráfico 4

México: relación entre distintos indicadores de bienestar, 2014

A. Indicadores IMBE y BLI

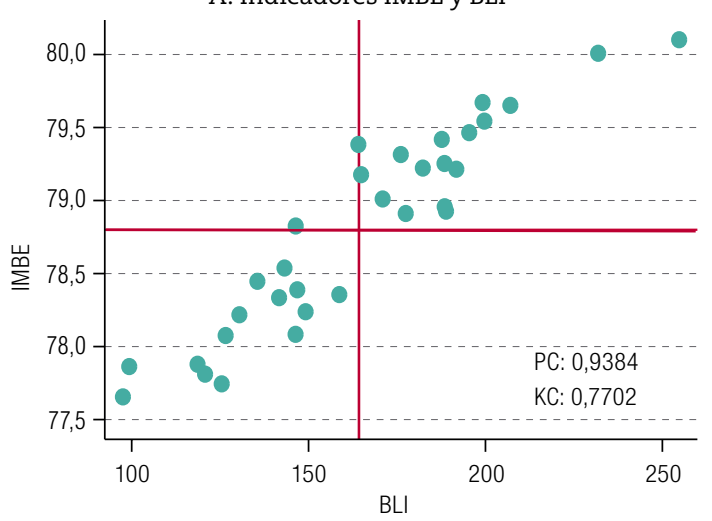

C. Indicadores IMBE y AMPI

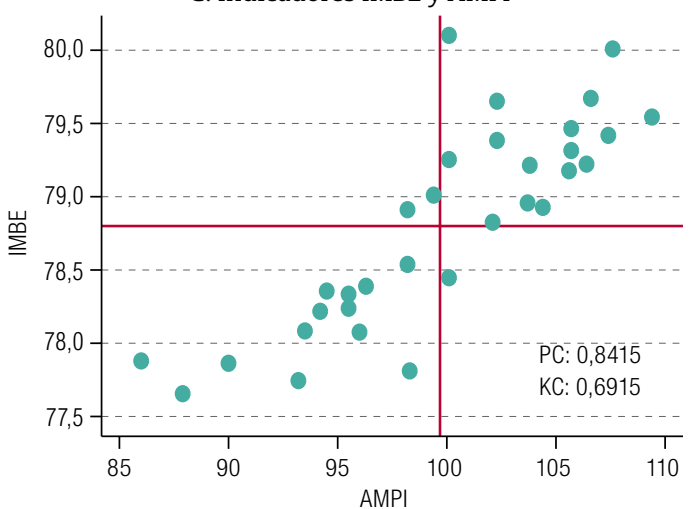

B. Indicadores IMBE y SoL

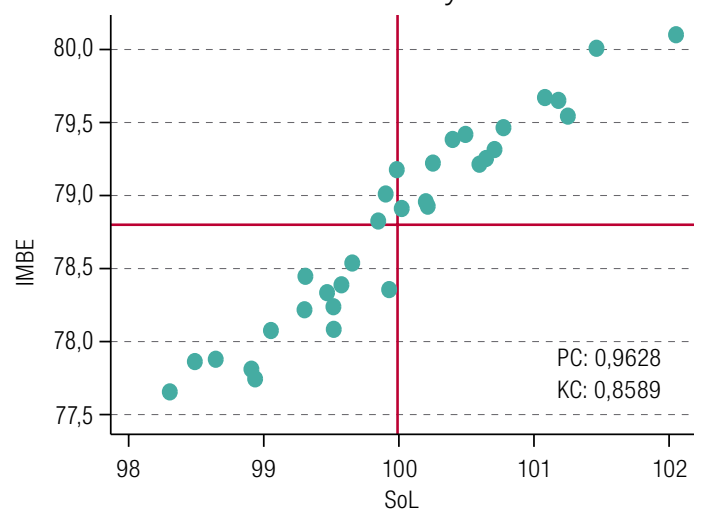

D. Indicadores BLI y AMPI

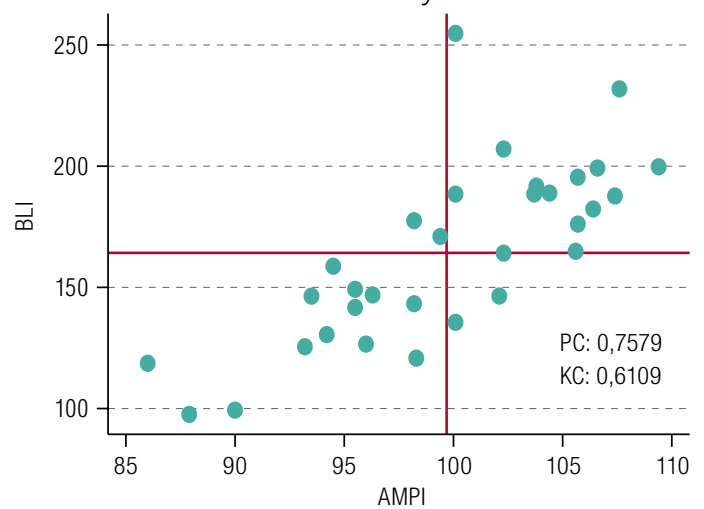

- Media del indicador

Fuente: Elaboración propia, sobre la base de datos del Instituto Nacional de Estadística y Geografía (INEGl) y la Organización de Cooperación y Desarrollo Económicos (OCDE).

Nota: IMBE: índice multidimensional de bienestar estatal; BLI: índice de bienestar basado en el Índice para una Vida Mejor de la OCDE (2015); SoL: índice de bienestar basado en los estándares de vida de Bérenger y Verdier-Chouchane (2007); AMPI: índice de Mazziota-Pareto ajustado, informado para 2014 por la OCDE (2015).

PC: correlación de Pearson; KC: correlación tau de Kendall.

Al comparar los indicadores con el AMPI, como se presenta en el gráfico 4, se observa que aunque las variaciones positivas en alguno de ellos están relacionadas con variaciones positivas en el otro, hay una diferencia importante en el posicionamiento que establecen respecto al bienestar de las entidades federativas; incluso el índice $B L I_{i}$, que comprende prácticamente los mismos datos que el índice AMPI, muestra diferencias considerables, al permitir distintas ponderaciones de indicadores y dimensiones en el bienestar total. El caso más notorio es el de Ciudad de México, que en todos los indicadores ocupa la posición número 1 de bienestar, excepto en el AMPI y el $B L I_{i}$, en el que se ubica cerca de la media (posiciones 15 y 7 , respectivamente). De hecho esta diferencia en los indicadores de bienestar de Ciudad de México es la que "penaliza" la correlación en los gráficos 4C y 4D; la diferencia se explica por el hecho de que esta entidad federativa se encuentra clasificada en los lugares más bajos en las dimensiones de bienestar subjetivo, específicamente en los indicadores de satisfacción con la vida y equilibrio entre trabajo y vida, y puesto que estas dimensiones tienen mayor relevancia en el AMPI que en el $I M B E_{i}$ o el $B L I_{i}$, el valor de bienestar global de Ciudad de México es mucho menor en el caso del AMPI. Esta diferencia evidente entre el $B L I_{i}$ y el AMPI, así como la similitud entre el $B L I_{i}$ y el $I M B E_{i}$, confirman la importancia de considerar la sensibilidad de la ponderación en la construcción de indicadores (Stiglitz, Sen y Fitoussi, 2009), incluso más que la elección de las variables, al menos tratándose de estos indicadores referentes al bienestar. 


\section{Conclusiones}

Al considerar que por su producto interno bruto (PIB) México es la decimoquinta mayor economía del mundo (según datos del Banco Mundial de 2015) y, a la vez, que más de la mitad de sus habitantes se encuentran en situación de pobreza, se advierte la complejidad de establecer políticas orientadas a aumentar el nivel de bienestar, por lo menos en el corto plazo, en el que es evidente el conflicto entre el crecimiento económico y la superación de los niveles de pobreza y desigualdad (López, 2004). En el mismo sentido, es indiscutible el argumento de que una política pública orientada a mejorar el bienestar de la población que considere de manera aislada sus componentes será limitada o infructuosa. Por ello, la necesidad de considerar diversos aspectos objetivos y subjetivos en la construcción de indicadores de bienestar es ineludible, de acuerdo con la teoría; al respecto, Stiglitz, Sen y Fitoussi (2009) hacen el aporte más relevante concerniente a recomendaciones teóricas y prácticas para la construcción de estos indicadores.

A pesar de las complicaciones adicionales concernientes a la medición de los aspectos subjetivos del bienestar, recientemente en México se han logrado avances importantes mediante el proyecto referente al bienestar autorreportado del INEGI, que ha permitido no solo obtener información sobre el bienestar general de la población a nivel nacional y estatal, sino además indagar sobre las relaciones que existen entre las distintas dimensiones del bienestar. Gracias a esta información, se han logrado también avances importantes en la construcción de indicadores de bienestar global más allá del índice de desarrollo humano o de los indicadores de pobreza del Consejo Nacional de Evaluación de la Política de Desarrollo Social (CONEVAL); entre ellos, el más reciente es el de la OCDE (2015), que, al abarcar 35 indicadores relativos a 12 dimensiones del bienestar, considera información compleja y vasta respecto a la situación de bienestar global de la población mexicana. Sin embargo, este indicador no concede importancia a la ponderación de cada dimensión en el nivel de bienestar global, tampoco utiliza indicadores de bienestar subjetivo representativos a nivel estatal y otorga poca relevancia a la desigualdad, lo que cuestiona la conveniencia de tomar decisiones sobre la base de su información. También a nivel individual es razonable afirmar que las distintas facetas del bienestar pueden diferir en importancia, pues es evidente la heterogeneidad imperante en la población mexicana en cuanto a ingresos, educación, acceso a servicios, tiempo dedicado a actividades laborales, ocio o cultura, entre otros aspectos. Incluso cabe la posibilidad de atribuir cierta validez a la postura maslowiana, en el sentido de ponderar primordialmente las dimensiones materiales del bienestar, como el hecho de contar con alimentos o salud, antes que, por ejemplo, otras dimensiones como las relacionadas con la participación en algún grupo social o el hecho de vivir en un contexto con un menor nivel de corrupción.

Por esta razón, en el presente estudio se adapta un índice de bienestar multidimensional para atender a las debilidades identificadas en el indicador de la OCDE, sobre la base de las recomendaciones de Stiglitz, Sen y Fitoussi (2009). Al utilizar una metodología meramente estadística, se evita la crítica de emitir juicios de valor respecto a la importancia relativa de cada dimensión, pero al mismo tiempo se hace posible que exista tal diferenciación en su ponderación. Además, el hecho de considerar los enfoques que proporcionan otros estudios (Bérenger y Verdier-Chouchane, 2007; OCDE, 2015) permite analizar la importancia de incluir ponderaciones, dimensiones e indicadores diferentes en la construcción del índice de bienestar. En este sentido, los resultados muestran que las mayores diferencias se presentan al otorgar distintas ponderaciones a las dimensiones, lo que respalda el argumento de que estas inciden de manera diferente en el bienestar; incluso la posibilidad de variar las ponderaciones es más importante que la misma elección de indicadores o dimensiones.

Otro aspecto relevante que resulta notorio es la importancia relativa que tuvieron las dimensiones e indicadores relacionados con el bienestar material, pues en todos los casos fue mayor que la de aquellos relacionados con el bienestar subjetivo; en este sentido, puede argumentarse que estos 
resultados resaltan la validez del índice de desarrollo humano como indicador de bienestar de la población mexicana. Aun con ello, esto no implica que el bienestar subjetivo no sea importante en el análisis, pero quizá en el caso mexicano el bienestar material esté mayormente relacionado con otras dimensiones del bienestar y, por tanto, sea prioritario atenderlo. Asimismo, es importante notar que, aunque se lleva a cabo un análisis de correlación, no se aborda el tema de la causalidad entre las dimensiones del bienestar y, por tanto, no se pueden obtener conclusiones sobre si los indicadores relacionados con la dimensión de bienestar material (como el ingreso) tienen un efecto positivo en el bienestar subjetivo. Por lo pronto, quizá en un país donde aproximadamente la mitad de la población padece algún tipo de pobreza sea más conveniente contar con un indicador de bienestar que pondere más la escasez de bienes materiales.

Otro aspecto que se debe considerar es que, a fin de permitir una comparación entre entidades federativas, como punto de partida para cada estado se pondera de la misma forma cada dimensión y, con el mismo argumento sobre la heterogeneidad expuesto anteriormente, esto puede presentarse como una limitante de este estudio, pues es de esperarse que existan diferencias regionales y estatales; por ejemplo, puede argumentarse que la población de los estados del sur obtiene un bienestar asociado a los indicadores ambientales diferente del que obtiene la población que vive en zonas donde los recursos naturales son escasos, como Ciudad de México o los estados del norte. Al respecto, un análisis a nivel de microdatos podría dar información concerniente a la existencia de diferencias en la ponderación de las dimensiones por cada entidad federativa, pero eso queda fuera del alcance de este estudio.

\section{Bibliografía}

Aiginger, K. y M. Firgo (2015), "Regional competitiveness under new perspectives", Policy Paper, № 26, Viena, WWW for Europe.

Arita, B. (2005), "La capacidad y el bienestar subjetivo como dimensiones de estudio de la calidad de vida", Revista Colombiana de Psicología, vol. 14, Bogotá, Universidad Nacional de Colombia.

Beckley, H. (2002), "Capability as opportunity: how Amartya Sen revises equal opportunity", Journal of Religious Ethics, vol. 30, № 1, Hoboken, Wiley.

Bentham, J. (2000), An Introduction to the Principles of Morals and Legislation, Kitchener, Batoche Books.

Benvin, E., E. Rivera y V. Tromben (2016), "Propuesta de un indicador de bienestar multidimensional de uso del tiempo y condiciones de vida aplicado a Colombia, el Ecuador, México y el Uruguay", Revista de la CEPAL, № 118 (LC/G.2676-P), Santiago, Comisión Económica para América Latina y el Caribe (CEPAL).

Bérenger, V. y A. Verdier-Chouchane (2007), "Multidimensional measures of well-being: standard of living and quality of life across countries", World Development, vol. 35, № 7, Amsterdam, Elsevier.

Bonini, A. (2008), "Cross-national variation in individual life satisfaction: effects of national wealth, human development, and environmental conditions", Social Indicators Research, vol. 87, N2 2, Nueva York, Springer.

Chakravarty, S. y M. Lugo (2016), "Multidimensional indicators of inequality and poverty", The Oxford Handbook of Well-being and Public Policy, M. Adler y M. Fleurbaey (eds.), Nueva York, Oxford University Press.

Graham, J. (2006), "Congeneric and (essentially) tau-equivalent estimates of score reliability: what they are and how to use them", Educational and Psychological Measurement, vol. 66, Nㅜ 6, Thousand Oaks, SAGE.

Hicks, D. (2002), "Gender, discrimination, and capability: insights from Amartya Sen", The Journal of Religious Ethics, vol. 30, № 1, Hoboken, Wiley.

Kaiser, H. (1974), "An index of factor simplicity", Psychometrika, vol. 39, № 1, Nueva York, Springer.

Kolm, S. (1997), Justice and Equity, Cambridge, MIT Press.

Krauss, A. y C. Graham (2013), "Subjective wellbeing in Colombia: some insights on vulnerability, job security, and relative incomes", Policy Research Working Paper, № 6672, Washington, D.C., Banco Mundial.

Lever, J. (2004), "Poverty and subjective well-being in Mexico", Social Indicators Research, vol. 68, No. 1, Nueva York, Springer.

López, J. (2004), "Pro-growth, pro-poor: is there a trade-off?", Policy Research Working Paper, № 3378, Washington, D.C., Banco Mundial. 
México, Gobierno de (2013), Plan Nacional de Desarrollo 2013-2018, Ciudad de México.

Nafziger, E. (2005), Economic Development, Cambridge, Cambridge University Press.

OCDE (Organización de Cooperación y Desarrollo Económicos) (2015), Measuring Well-being in Mexican States, París, OECD Publishing.

(2014), How's Life in Your Region? Measuring Regional and Local Well-being for Policy Making, París, OECD Publishing.

(2008), Handbook on Constructing Composite Indicators. Methodology and User Guide, París, OECD Publishing.

Plata, L. (1999), “Amartya Sen y la economía del bienestar”, Estudios Económicos, vol. 14, N 1, Ciudad de México, El Colegio de México.

Rawls, J. (1971), A Theory of Justice, Cambridge, Harvard University Press.

Rojas, M. (2007), "The complexity of well-being: a life-satisfaction conception and a domains-of-life approach", Well-being in Developing Countries: from Theory to Research, I. Gough y J. McGregor (eds.), Nueva York, Cambridge University Press.

(2005), “¿Qué es desarrollo económico?”, La formación de economistas: ensayos en honor de Pepita Echandi, J. Vargas e Y. Xirinachs (eds.), San José, Universidad de Costa Rica.

Sen, A. (1984), Resources, Values, and Development, Cambridge, Harvard University Press.

StataCorp (2013), Stata Multivariate Statistics Reference Manual: Release 13, College Station, StataCorp LP.

Stiglitz, J., A. Sen y J. Fitoussi (2009), Report by the Commission on the Measurement of Economic Performance and Social Progress, París, The Commission.

Tavakol, M. y R. Dennick (2011), "Making sense of Cronbach's alpha", International Journal of Medical Education, vol. 2, Nottingham, Mayfair Publications.

Thomson, W. (2016), "Fair allocation", The Oxford Handbook of Well-being and Public Policy, M. Adler y M. Fleurbaey (eds.), Nueva York, Oxford University Press.

Valencia, G. y J. Cuervo (1999), "Crítica a las bases éticas de la teoría neoclásica en la propuesta del bienestar social de Amartya Sen”, Lecturas de Economía, № 51, Medellín, Universidad de Antioquia.

Veenhoven, R. (2005), "Is life getting better? How long and happy people live in modern society", European Psychologist, vol. 10, $\mathrm{N}^{\circ}$ 4, Boston, Hogrefe.

Villatoro, P. (2012), "La medición del bienestar a través de indicadores subjetivos: una revisión”, serie Estudios Estadísticos y Prospectivos, № 79 (LC/L.3515), Santiago, Comisión Económica para América Latina y el Caribe (CEPAL).

Wells, T. (2016), "Sen's capability approach", Internet Encyclopedia of Philosophy, J. Fieser y B. Dowden (eds.), Martin, University of Tennessee at Martin.

Yurdugül, H. (2008), "Minimum sample size for Cronbach's coefficient alpha: a Monte-Carlo study”, Hacettepe University Journal of Education, vol. 35, Beytepe, Hacettepe University. 


\section{Anexo A1}

El manual para la construcción de indicadores compuestos de la OCDE (2008) contempla diez pasos: i) elaboración del marco teórico, ii) selección de datos, iii) tratamiento de datos perdidos, iv) análisis multivariado, v) normalización, vi) ponderación y agregación, vii) análisis de sensibilidad, viii) escrutinio de datos, ix) vínculo a otros indicadores y $\mathrm{x}$ ) visualización de resultados. En este artículo se abordan primordialmente las recomendaciones técnicas de los pasos cuarto y sexto concernientes al análisis de factores de componentes principales y el alfa de Cronbach.

La OCDE (2008) menciona que el alfa de Cronbach no es una prueba estadística sino un coeficiente de confiabilidad (estabilidad interna) basado en la correlación entre indicadores individuales. De esta forma, una correlación alta señala que los indicadores individuales miden la misma variable o dimensión subyacente, en este caso el bienestar. Cabe destacar que a pesar de que el cálculo del alfa de Cronbach permite obtener el valor de una escala para cada observación, utilizar esta escala como la variable o dimensión subyacente puede llevar a conclusiones erróneas. Al respecto algunos autores (por ejemplo, Graham (2006) y Tavakol y Dennick (2011)) enfatizan que el alfa de Cronbach no debe considerarse como una prueba de unidimensionalidad, pues puede ser engañoso si la escala subyacente contempla más de una dimensión. Por tanto, Tavakol y Dennick (2011) sugieren utilizar el análisis de factores para identificar más de una dimensión en una prueba.

Así, considerando que la escala obtenida por el alfa de Cronbach sea multidimensional, se sigue la sugerencia de Tavakol y Dennick (2011) y OCDE (2008) de utilizar el análisis de factores de componentes principales para construir el indicador de bienestar conforme a los ponderadores obtenidos mediante dicho método; aunque existen otros enfoques como el axiomático y el de la teoría de conjuntos difusos (Chakravarty y Lugo, 2016), el análisis de factores de componentes principales es de fácil aplicación y ofrece ventajas en cuanto a la interpretación de sus resultados. Para ello, la OCDE (2008) afirma que es necesario que exista una correlación relativamente alta entre las variables originales $x_{i j}$; de otro modo, ni siquiera tendría sentido su aplicación. Por lo tanto, después de calcular el alfa de Cronbach, se realiza un análisis de correlaciones entre las variables $x_{i j}$ para confirmar que las relaciones sean estadísticamente significativas y tengan el signo esperado, e indagar sobre los grupos de variables con mayor relación que pudieran encontrarse al momento de realizar el análisis de factores.

Después de confirmar la existencia de correlaciones significativas entre las variables de interés, se realiza el análisis de factores de componentes principales para obtener $W_{k}$ y $w_{j k}$, utilizados en la ecuación (1), y su validación mediante la medida de Kaiser-Meyer-Olkin. De acuerdo con el criterio de la OCDE (2008), se consideran los factores o componentes principales que cumplan con tres características: i) que sean ortogonales y contribuyan a la varianza total con al menos un $10 \%$; ii) que contengan la mayor proporción de varianza explicada con un autovalor mayor que 1, y iii) que tengan una contribución acumulada de la varianza total de al menos un $60 \%$. Posteriormente, se obtienen los ponderadores a partir de las cargas de factores al cuadrado normalizadas por la varianza del factor. Estos ponderadores normalizados se obtienen de los factores sometidos a rotación mediante el método varimax para minimizar el número de indicadores en cada factor y tener una estructura más simple en la composición de los factores utilizados; en otras palabras, mediante este método de rotación varimax se transforman las cargas originales (sin rotación) sin afectar la varianza de los factores para mejorar su interpretación (StataCorp, 2013) y facilitar los cálculos de la ecuación (1). 